US Army Corps

of Engineers ${ }_{\circledast}$

Engineer Research and

Development Center

Ecosystem Management and Restoration Research ProgramModeling and Decision Support

Monitoring Ecological Restoration with Imagery Tools (MERIT): Python-based Decision Support Tools Integrated into ArcGIS for Satellite and UAS Image Processing, Analysis, and Classification

Kristofer Lasko and Sean Griffin

April 2021 
The US Army Engineer Research and Development Center (ERDC) solves the nation's toughest engineering and environmental challenges. ERDC develops innovative solutions in civil and military engineering, geospatial sciences, water resources, and environmental sciences for the Army, the Department of Defense, civilian agencies, and our nation's public good. Find out more at www.erdc.usace.army.mil.

To search for other technical reports published by ERDC, visit the ERDC online library at https://erdclibrary.on.worldcat.org/discovery. 


\section{Monitoring Ecological Restoration with Imagery Tools (MERIT): Python-based Decision Support Tools Integrated into ArcGIS for Satellite and UAS Image Processing, Analysis and Classification}

Ecosystem Management and Restoration Research Program (EMRRP)-Modeling and Decision Support

Kristofer Lasko and Sean Griffin

Geospatial Research Laboratory

US Army Engineer Research and Development Center

7701 Telegraph Road

Alexandria, VA 22315-3864

Final Report

Approved for public release; distribution is unlimited.

Prepared for Headquarters, US Army Corps of Engineers

Washington, DC 20314-1000

Under PE A1120, Project 485296 "Using UAS and Satellite Imagery to map aquatic and terrestrial Ecosystems" 


\section{Abstract}

Monitoring the impacts of ecosystem restoration strategies requires both short-term and long-term land surface monitoring. The combined use of unmanned aerial systems (UAS) and satellite imagery enable effective landscape and natural resource management. However, processing, analyzing, and creating derivative imagery products can be time consuming, manually intensive, and cost prohibitive. In order to provide fast, accurate, and standardized UAS and satellite imagery processing, we have developed a suite of easy-to-use tools integrated into the graphical user interface (GUI) of ArcMap and ArcGIS Pro as well as open-source solutions using NodeOpenDroneMap. We built the Monitoring Ecological Restoration with Imagery Tools (MERIT) using Python and leveraging third-party libraries and open-source software capabilities typically unavailable within ArcGIS. MERIT will save US Army Corps of Engineers (USACE) districts significant time in data acquisition, processing, and analysis by allowing a user to move from image acquisition and preprocessing to a final output for decision-making with one application. Although we designed MERIT for use in wetlands research, many tools have regional or global relevancy for a variety of environmental monitoring initiatives.

DISCLAIMER: The contents of this report are not to be used for advertising, publication, or promotional purposes. Citation of trade names does not constitute an official endorsement or approval of the use of such commercial products. All product names and trademarks cited are the property of their respective owners. The findings of this report are not to be construed as an official Department of the Army position unless so designated by other authorized documents. 


\section{Contents}

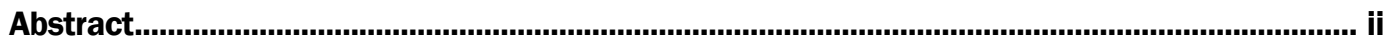

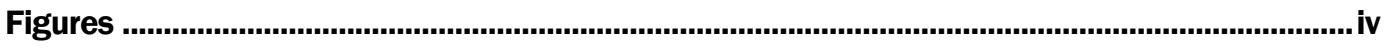

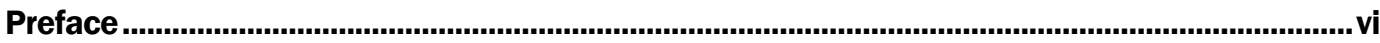

1 Introduction.............................................................................................................................. 1

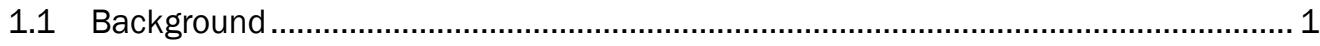

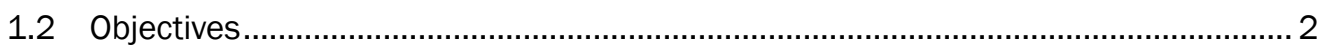

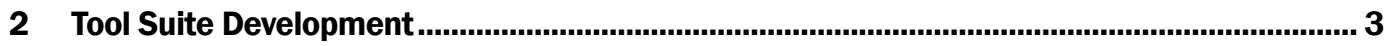

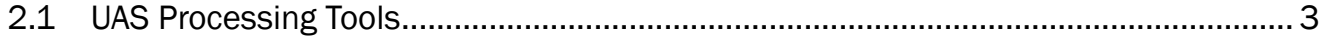

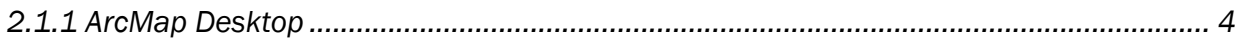

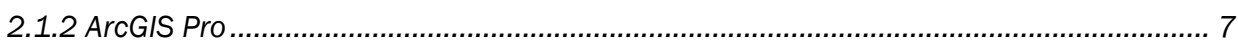

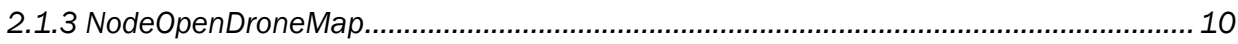

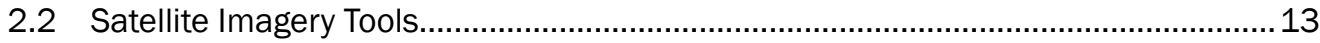

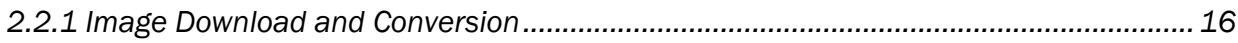

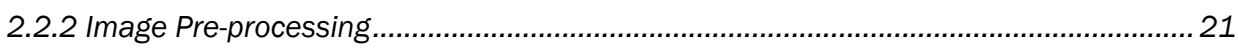

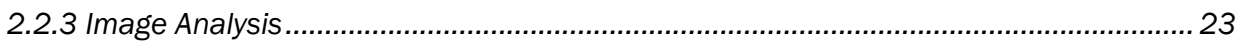

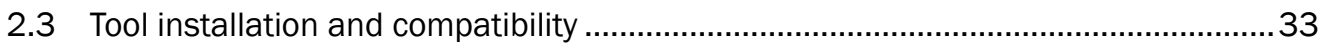

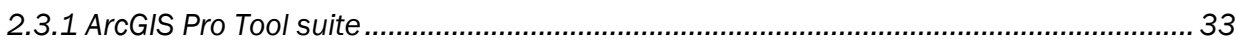

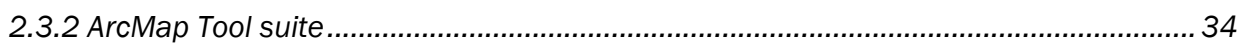

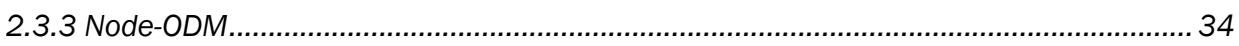

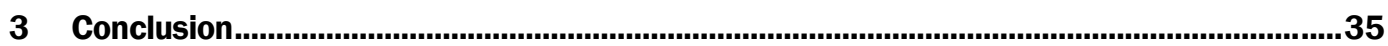

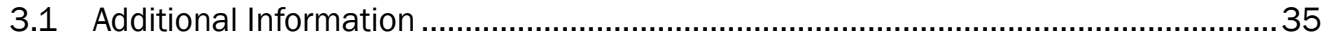

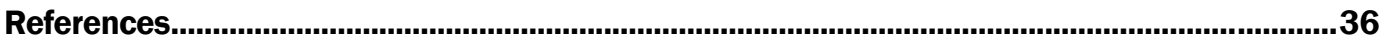

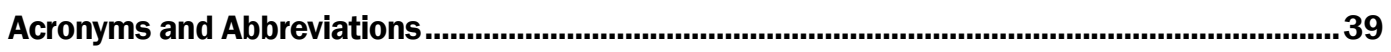

Report Documentation Page 


\section{Figures}

Figure 1. Conceptual model of the Ecosystem Management and Restoration

Research Program (EMRRP) Monitoring Ecological Restoration with Imagery Tools (MERIT) unmanned aerial systems (UAS) subtools. There are three different sets: (1) one set that exclusively uses open-source capabilities without a need for ArcGIS licenses, (2) an ArcMap version compatible with ArcMap 10.6 or higher, and (3) a workflow within ArcGIS Pro's Image Analyst Extension.

Figure 2. The UAS Workflow ArcToolbox for processing UAS imagery and derived products

Figure 3. Step one of the UAS Workflow is to create a mosaic database to store image frames.

Figure 4. Step two of the UAS Workflow computes the camera model, applies block adjustments, and creates an orthomosaic.

Figure 5. Step three of the UAS Workflow generates point clouds and terrain models from the stereo model.

Figure 6. UAS Workflow from raw imagery to spatial products, which include colorbalanced orthomosaic, point cloud, digital terrain models (DTM), and digital surface models (DSM). DTM and color-balanced orthomosaic are shown here

Figure 7. ArcGIS Pro Ortho Workspace showing image collection, spatial reference, and camera model, in this case a Ricoh GR I.

Figure 8. Contents pane and Map View showing camera locations, flight path, and source data information.

Figure 9. Ortho Mapping menu providing refinement and ortho product options. 10

Figure 10. NodeODM Manager displaying local REST API session in Chrome browser. 11

Figure 11. Final orthomosaic and digital surface model produced by NodeODM (Fort AP Hill). Top right: colorized DSM; bottom right: wetland zone.

Figure 12. Colorized point-cloud image displayed by height.

Figure 13. Layout of MERIT in ArcGIS Pro showcasing the different sets of tools and associated organization structure.

Figure 14. Conceptual model of the satellite image processing and analysis tool suite with an example, likely processing workflow.

Figure 15. Screenshot of the image acquisition tool that directly downloads imagery in batches from ESA public-facing servers over a user's AOI. Tool message output is shown at the top right, highlighting that the user is informed of each step in the process as well as any errors. We recommend $20 \mathrm{~m}$ imagery because of increased spectral versatility and compatibility with automatic wetland classification tools.

Figure 16. High-level conceptual model showing how MERIT takes as input a folder of multiple Sentinel-2 images. Then the ArcGIS Pro tool calls the Sen2Cor atmospheric correction tool and converts the image to surface reflectance, as shown in the bottom two images.

Figure 17. Left-side images contain cloudy Sentinel-2 images. The right-side image is a composite that combines cloud-free pixels from both dates in order to create a cloud-reduced composite for subsequent analysis. 
Figure 18. Environmental indices derived from EMRRP MERIT. The top left images shows the custom graphical user interface (GUI) integrated into ArcGIS Pro and highlights that multiple images can be processed.

Figure 19. Ensemble classifier result for an example six-class, user-created image classification scheme with corresponding pixel agreement map. The agreement map shows the number of classifiers that agree on the resulting land cover type for a given pixel. The conceptual model is shown in the middle.

Figure 20. Example possible output for the regional statistics tools. The user inputs a shapefile of their Area of Interest (AOI), a time series of raster data, and outputs corresponding tables that can create temporal visualizations of an area. This example uses burned-area data; however, a variety of raster data can be used.

Figure 21. Two dates of Sentinel-2 imagery (leaf on, leaf off) are input into the tool to create a wetland area land cover type map. An example output is shown on the right. The user need only input their imagery; no training data is required.

Figure 22. Absolute change between two dates of NDVI in a wetland area of Virginia. Most change in this image is associated with seasonality. 


\section{Preface}

This study was conducted for the Geospatial Research Laboratory (GRL) under PE A1120, Project 485296, "EMRRP." The project manager at the time of writing was Dr. Trudy Estes of the Environmental Laboratory (EL).

The work was performed by the Data and Signature Analysis Branch (TRS) of the TIG Research Division (TR), U.S. Army Engineer Research and Development Center, Geospatial Research Laboratory (ERDC-GRL). At the time of writing, Ms. Jennifer L. Smith was Chief, TR-S; Mr. Jeffrey Murphy was Acting Chief, TR; and Mr. Vijay Acharya, TV-T was the Acting Technical Director for ERDC-GRL. The Deputy Director of ERDC-GRL was Ms. Valerie L. Carney and the Director was Mr. Gary Blohm.

COL Teresa A. Schlosser was Commander of ERDC, and Dr. David W. Pittman was the Director. 


\section{Introduction}

\subsection{Background}

Wetlands are one of the most critically important ecosystems across the world. Wetlands is a generic term that includes many types of aquatic and semiaquatic environments. Wetlands consist of freshwater, saltwater, or brackish water environments that may be tidally influenced. The general types of wetlands are marsh, bog, swamp, and fen. The common subtypes of wetlands in the United States include mangrove forests, freshwater swamp forests, and various marsh systems. Wetlands consist of strong biological productivity with incredibly biodiverse flora and fauna (Bedford et al. 2001). Wetlands often occur not as single units but as a collection of small patches within a heterogenous landscape-leaving many species isolated and vulnerable to extinction (Dodd 1992).

Not only are wetland areas biodiverse and vulnerable, but they also serve as vital natural filters of environmental contaminants and pollutants, natural flood barriers to human development, sources of ecotourism and recreation, as well as economically important for their natural resources (Haarstad 2012). Recent research indicates that mangrove forests form one of the major carbon sinks, with significant soil and vegetative biomass; however, these ecosystems are subject to increasing levels of destruction and degradation (Hamilton 2018). In the United States, wetland cover types have decreased significantly over recent decades. For example, Dahl 2011 found a decrease of 25,000 ha ${ }^{1}$ between 2004 and 2009. While wetlands only represent about $6 \%$ of land area in the United States (Homer 2020), they provide disproportionately important environmental and economic benefits.

The US Army Corps of Engineers (USACE) Ecosystem Management and Restoration Research Program (EMRRP) focuses on better understanding these vital ecosystems by improving our fundamental understanding of the physical and biological processes and interactions within wetland ecosystems. The research and development focus areas of EMRRP include multiobjective restoration, integrity and sustainability, inland resource management, coastal

1. For a full list of the spelled-out forms of the units of measure used in this document, please refer to US Government Publishing Office Style Manual, 31st ed. (Washington, DC: US Government Publishing Office, 2016), 248-52, https://www.govinfo.gov/content/pkg/GPO-STYLEMANUAL-2016/pdf/GPO-

STYLEMANUAL-2016.pdf. 
resilience and function, invasive species management, ecological infrastructure, and modeling and decision-support tools (emrrp.el.erdc.dren.mil/index.html), the latter representing the primary focus of this research project.

\subsection{Objectives}

The downloading, processing, and analyzing of satellite and UAS imagery for wetland area mapping and monitoring can be time consuming, non-standardized, and cost prohibitive. This report summarizes new geospatial decision support tools developed by GRL in order to (1) provide faster and standardized processing and analysis of satellite and UAS imagery for environmental analysis with emphasis on aquatic and terrestrial vegetation and (2) implement this processing capability in an easy-to-use ArcMap and ArcGIS Pro Python toolkit that facilitates transferability to USACE technicians, biologists, hydrologists, and other GIS users.

To demonstrate the utility of these algorithms and highlight the need for an EMRRP MERIT GIS tool suite to exploit them, a mapping scenario covering USACE-Jacksonville District monitoring sites at Woerner and Hog Island, Florida, was conducted as an earlier part of this project (Griffin and Lasko 2020). The study sites contain such land use categories as abandoned orange groves under active restoration into a predevelopment wetland ecosystem. Further details of this proof-of-concept study are provided here: http://dx.doi.org/10.21079/11681/38086. 


\section{Tool Suite Development}

MERIT enables GIS users to download, process, and analyze satellite and UAS imagery for faster, standardized wetland area mapping and monitoring. The tools are written using Python 3 in conjunction with third-party libraries, open-source command line tools, and docker containers. The tools are fully integrated into ArcGIS software with a graphical user interface (GUI), and the suite contains two sets of tools. The first set comprises the UAS processing tools that include an ArcToolbox for ArcMap Desktop 10.6 or greater, standard operating procedures for the Ortho Mapping Workspace in ArcGIS Pro, and open-source tools developed by OpenDroneMap. The second set comprises the satellite imagery tools designed for ArcGIS Pro (version 2.5), which offer bulk acquisition and processing capabilities with an emphasis on Sentinel-2 $20 \mathrm{~m}$ imagery including cloud compositing, conversion to surface reflectance, regional statistics, and automated image classification.

\subsection{UAS Processing Tools}

Applying overhead remote sensing as an instrument for natural resource protection and monitoring is not a new concept. The Landsat mission of civil sensors has routinely collected earth observation data since 1972 and was widely considered the routine sensor for environmental monitoring applications for decades (Cohen and Goward 2004).

Advances in remote sensing technology in recent years have significantly supplemented modern ecological applications with a myriad of sensors and platforms (Luque et al. 2018). One such technology is UAS, which now plays a greater role in environmental data collection and monitoring. As with any overhead imagery collection, processing forms a fundamental part of the data workflow. However, aerial platforms like UAS suffer from a range of geometric distortions caused by the pitch, roll, and yaw of the vehicle, in addition to the camera perspective. Furthermore, unlike scanning radiometers found on satellite sensors, UAS optical sensors collect frame imagery that requires specialized photogrammetric solutions to mosaic the hundreds of frames acquired during a typical collection as well as to correct for geometric and radiometric distortion (Tmusic et al. 2020).

Many commercial and open-source software solutions already exist for preprocessing UAS imagery, the most notable being Pix4D, Agisoft Metashape, and OpenDroneMap (ODM). Commercial software licensing fees, as well as the 
capabilities required to successfully implement these products, can prohibit their use. However, USACE districts have access to enterprise licensing of Esri ArcGIS software that now includes robust photogrammetric functions specific to aerial image raster processing, including a large database of camera models used widely by the UAS Community of Practice.

The proceeding sections detail the installation, application, and geospatial analysis products derived from custom ArcMap Desktop UAS image processing tools, standard operating procedures that describe the UAS Processing workflow available in ArcGIS Pro, and open-source ODM. Figure 1 shows a conceptual model of the different UAS capabilities.

Figure 1. Conceptual model of the Ecosystem Management and Restoration Research Program (EMRRP) Monitoring Ecological Restoration with Imagery Tools (MERIT) unmanned aerial systems (UAS) subtools. There are three different sets: (1) one set that exclusively uses open-source capabilities without a need for ArcGIS licenses, (2) an ArcMap version compatible with ArcMap 10.6 or higher, and (3) a workflow within ArcGIS Pro's Image Analyst Extension.

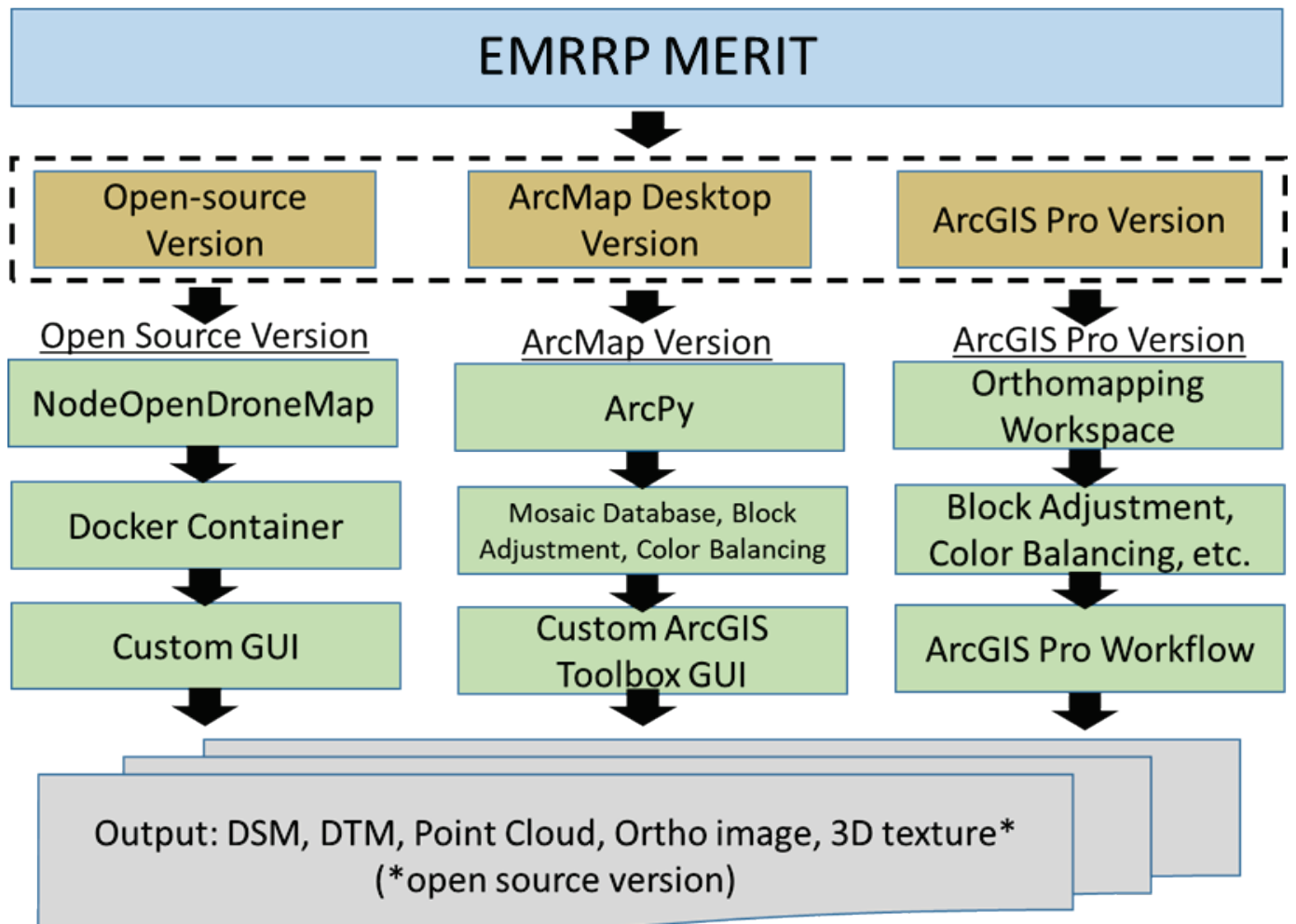

\subsubsection{ArcMap Desktop}

ArcMap Desktop uses the ArcPy site package to perform geographic data analysis, data conversion, data management, and map automation with the Python programming language. The latest versions of ArcMap Desktop 
(10.6 or greater) provide various ArcPy raster-processing functions, including support for adjusting UAS data and producing ortho products such as mosaics, passive point clouds, digital terrain models (DTM), and digital surface models (DSM). These products can be further analyzed to create very high spatial resolution land-cover maps or serve as an imagebased reference to train image classifiers for satellite imagery. However, processing UAS imagery in ArcMap Desktop requires a series of manually extensive steps. To facilitate this process, researchers at the Geospatial Research Laboratory (GRL) packaged the entire process into an ArcToolbox installer called the UAS Workflow (Figure 2).

Figure 2. The UAS Workflow ArcToolbox for processing UAS imagery and derived products.

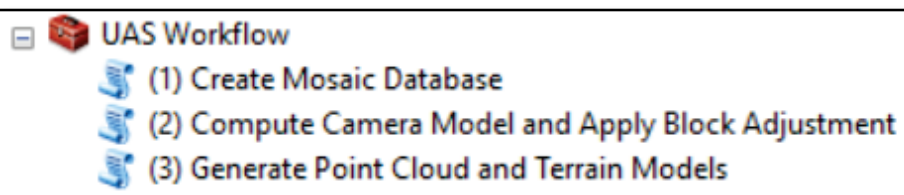

The process consists of three main steps. Step one creates a raster mosaic database that stores the UAS imagery and provides metadata on the coordinate system and raster type. Raster type is set to UAV/UAS as default, but this toolset can create a geodatabase for any raster type, including satellite imagery (Figure 3).

Figure 3. Step one of the UAS Workflow is to create a mosaic database to store image frames.

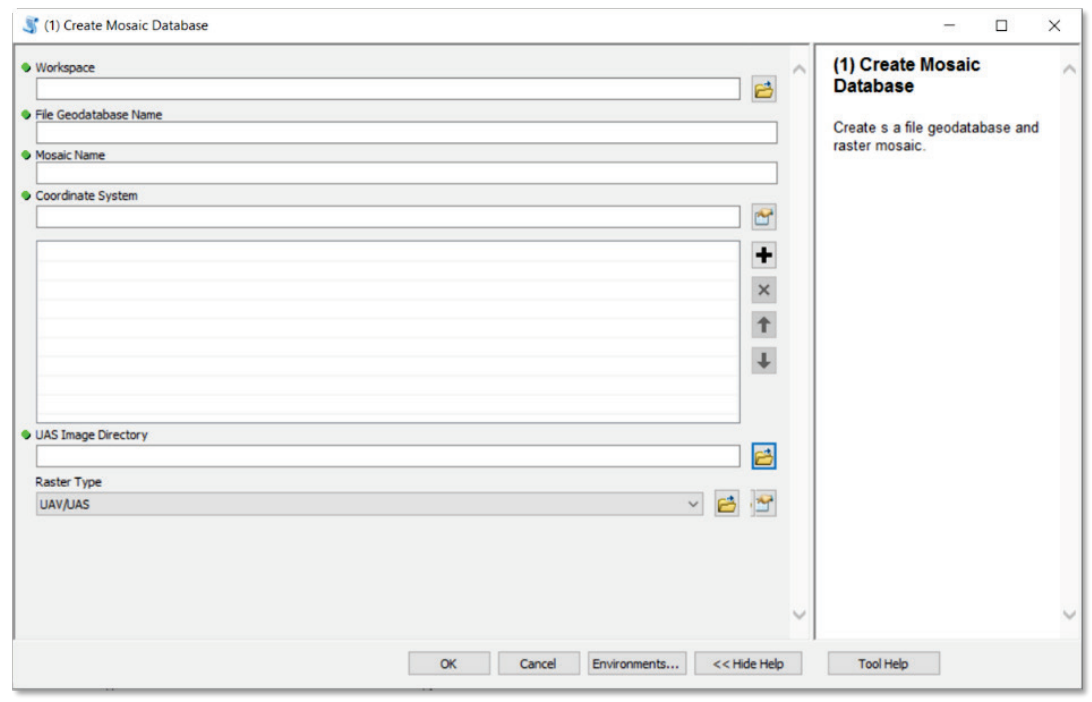


Step two loads the raster mosaic from the first step and reads the EXIF metadata from the UAS imagery. This step uses the ArcMap photogrammetry tools to perform rigorous block adjustment of the UAS mosaic. The output product is an orthorectified mosaic. The default camera model is set to Frame type (Figure 4).

Figure 4. Step two of the UAS Workflow computes the camera model, applies block adjustments, and creates an orthomosaic.

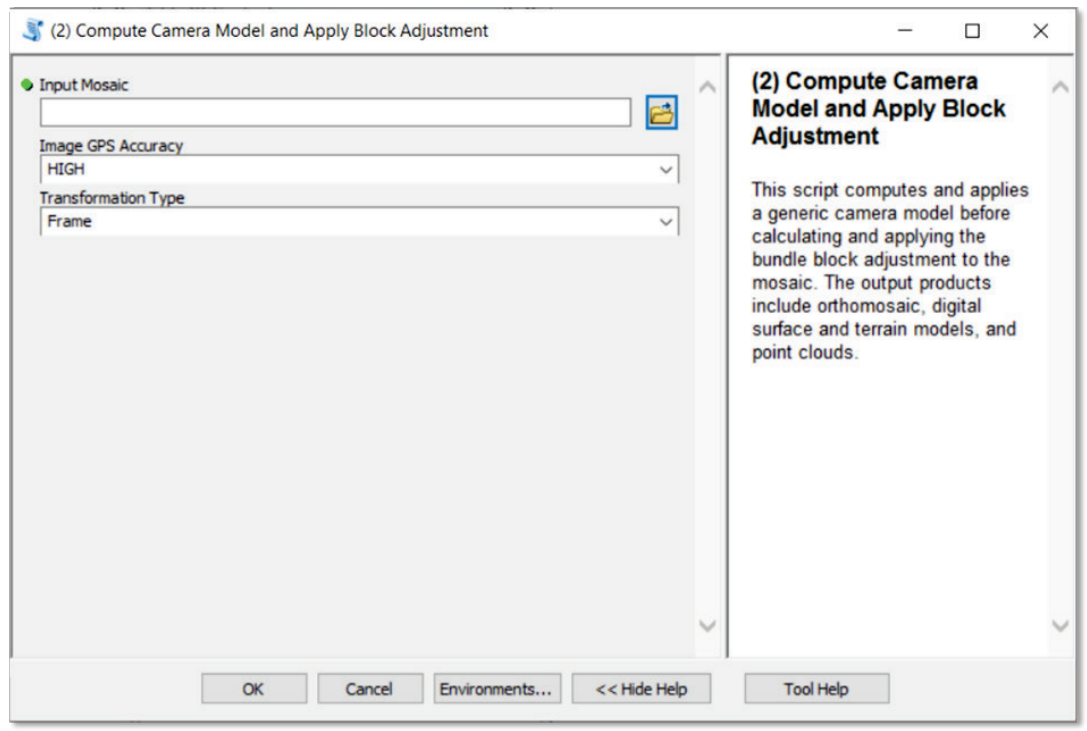

Step three is optional. This step applies photogrammetric techniques to the orthomosaic by creating a stereo model that can derive various terrain products, including digital terrain and surface models, and a passive point-cloud data set (Figure 5).

These terrain products can be used in conjunction with imagery to improve land-cover classification results. For example, a normalized digital surface model (NDSM) could be fused with imagery to help separate spectrally similar vegetation types by exploiting height differences. 
Figure 5. Step three of the UAS Workflow generates point clouds and terrain models from the stereo model.

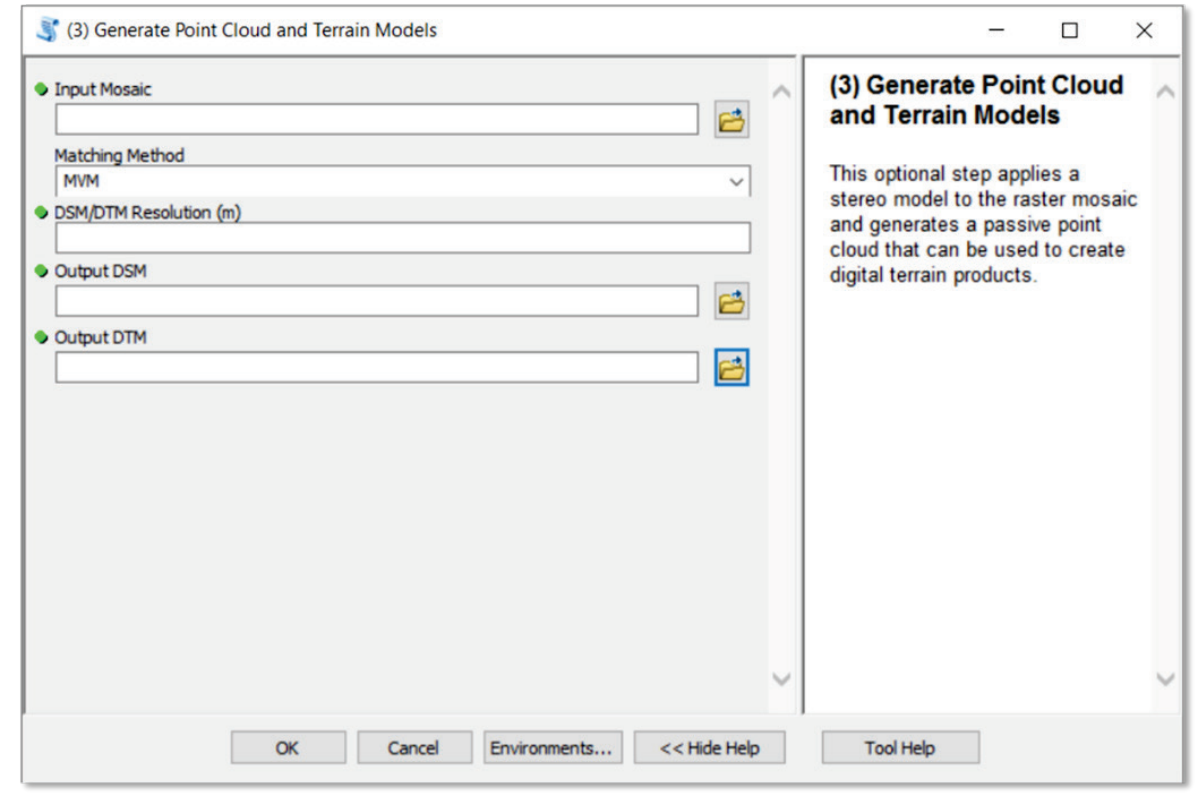

Figure 6 illustrates the UAS Workflow and final products generated from a data set collected over a reservoir in Colorado, USA.

Figure 6. UAS Workflow from raw imagery to spatial products, which include color-balanced orthomosaic, point cloud, digital terrain models (DTM), and digital surface models (DSM). DTM and color-balanced orthomosaic are shown here.

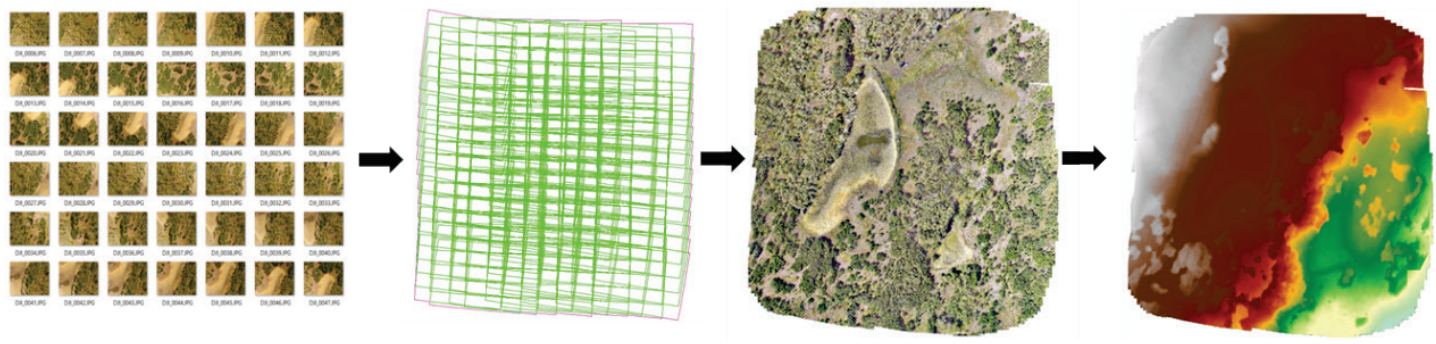

\subsubsection{ArcGIS Pro}

ArcGIS Pro offers significantly higher UAS processing performance compared to ArcMap Desktop through the Ortho Mapping Workspace (OMW). The OMW provides the user with built-in tools to photogrammetrically correct imagery from drones, satellites, digital photography, and scanned aerial photography by removing geometric distortion induced by sensor, platform, and terrain displacement. OMW produces a seamless orthomosaic by using edge matching and color balancing. Similar to the UAS Workflow tool for ArcMap Desktop, the resulting orthomosaic can generate DTM, DSM, and point clouds. In 
addition, ArcGIS Pro provides a true 64-bit architecture with parallel processing and graphics processing unit compute capability that significantly reduces processing times.

The workspace starts with adding a folder containing the UAS image frames and designating the raster type as drone, which provides a sensor drop-down list that includes Generic, RedEdge, and Altum. This step also initiates geolocation extraction from the EXIF metatags, spatial reference, and the camera model (Figure 7).

Figure 7. ArcGIS Pro Ortho Workspace showing image collection, spatial reference, and camera model, in this case a Ricoh GR I.

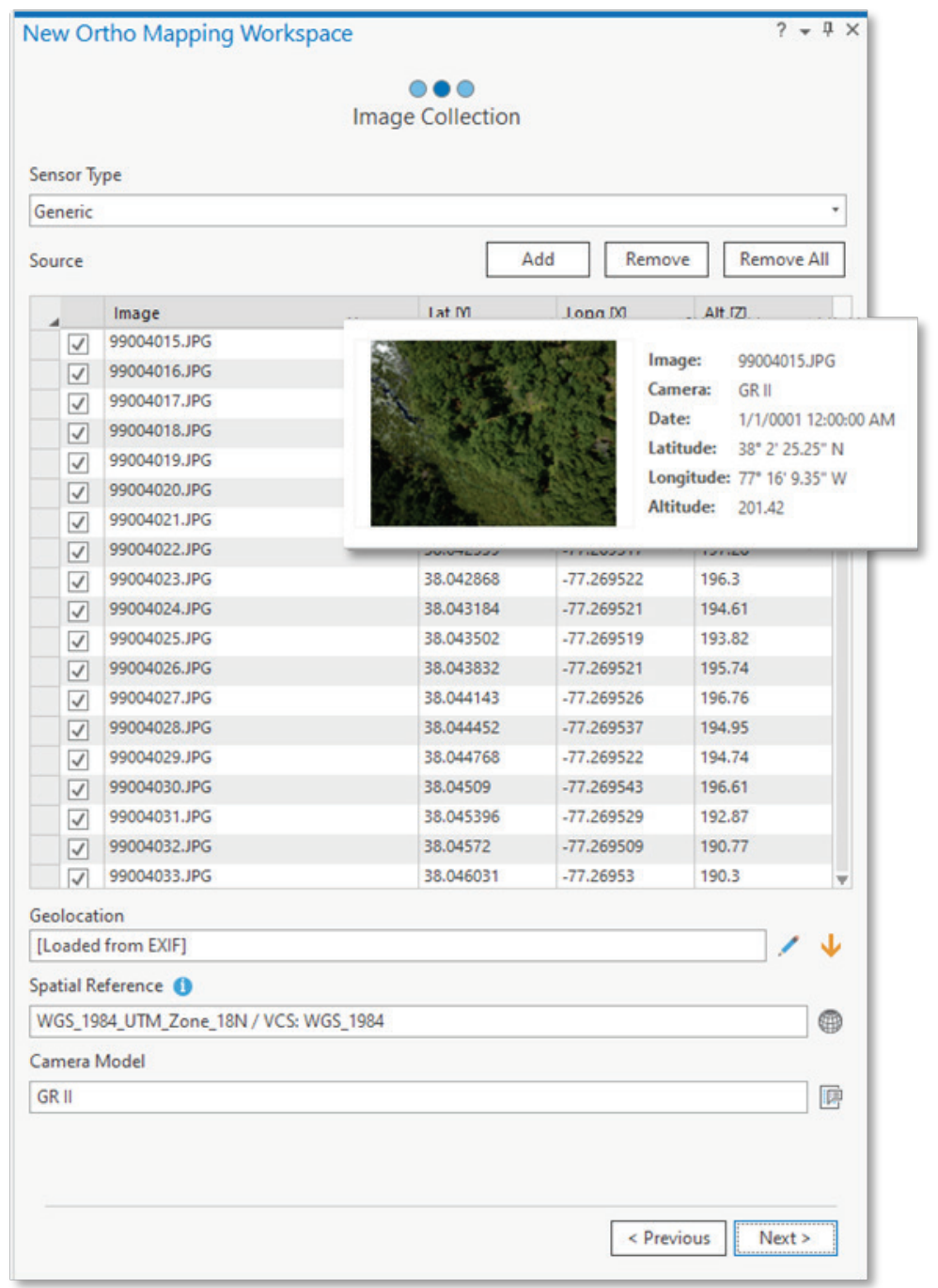


When the OMW is created, an Ortho Mapping category is populated in the Catalog pane. The Contents pane will also contain a variety of layers associated with the workspace, such as flight and source data, control points, solution points, and data products (Figure 8).

Figure 8. Contents pane and Map View showing camera locations, flight path, and source data information.

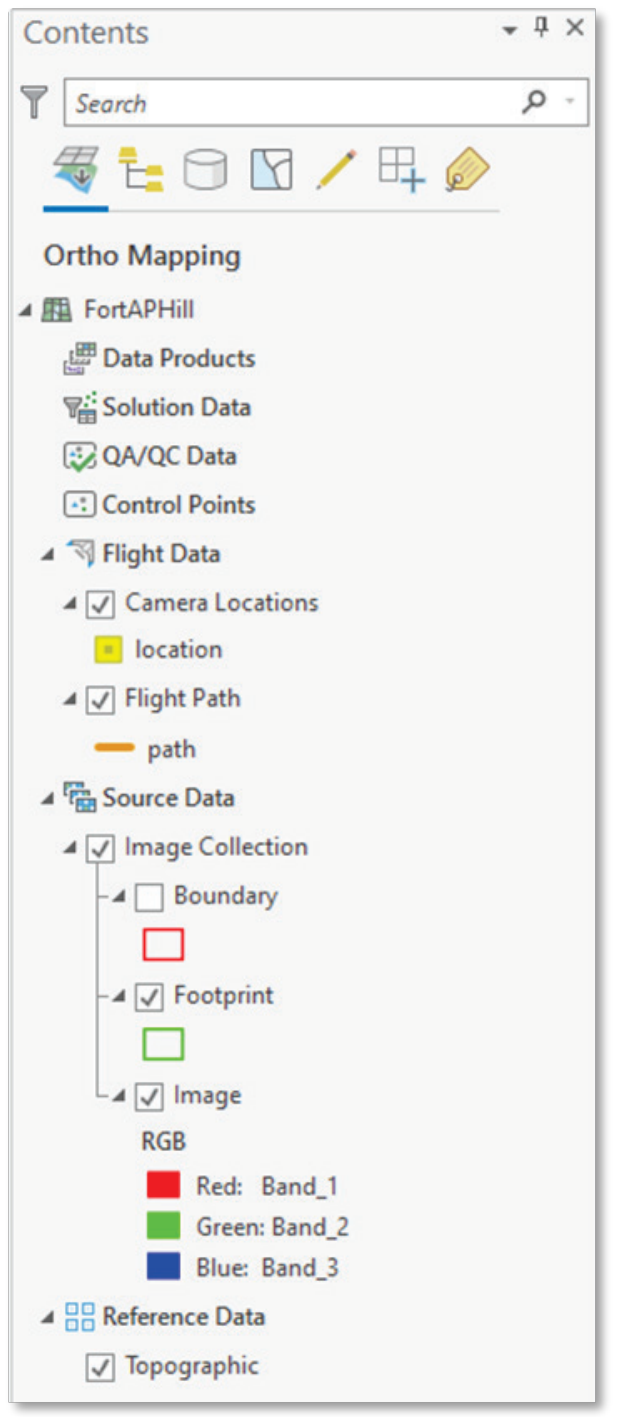

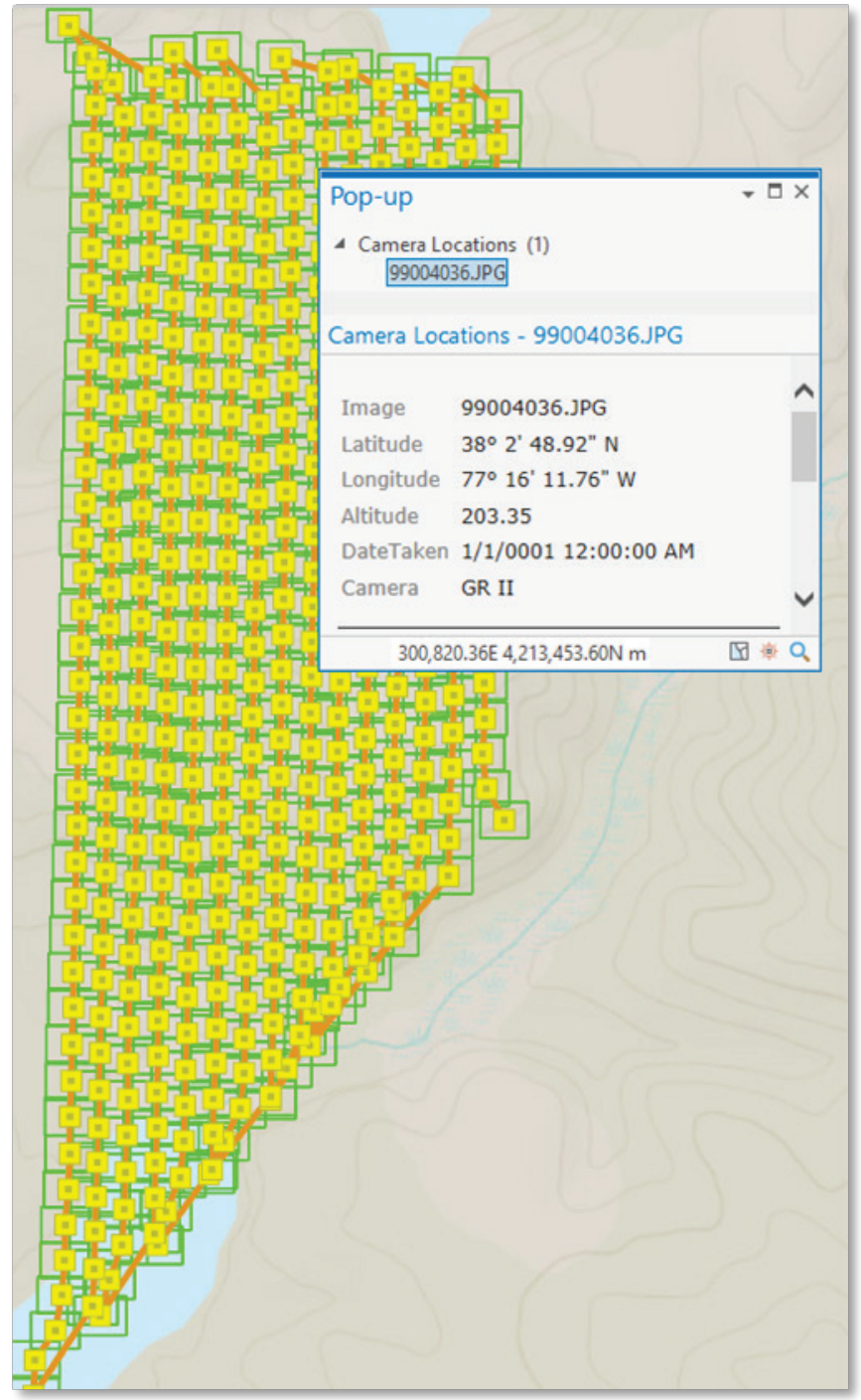

When the process is complete, the Ortho Mapping menu provides a series of options that includes setting the block adjustments, editing and refining GCPs, and producing ortho products (Figure 9). 
Figure 9. Ortho Mapping menu providing refinement and ortho product options.

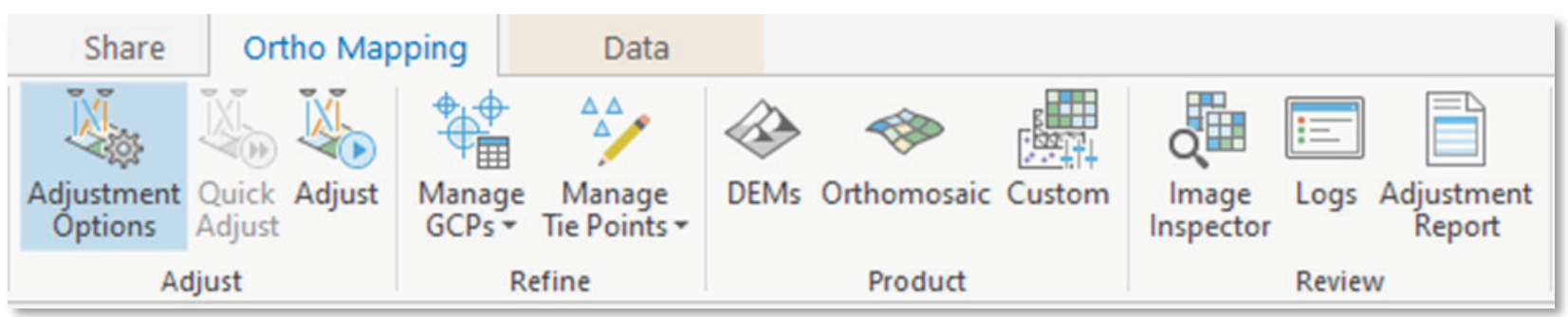

\subsubsection{NodeOpenDroneMap}

NodeOpenDroneMap, or NodeODM, is an open-source drone mapping software with extendable application and API for UAS image processing. Similar to the Esri commercial options, NodeODM generates georeferenced orthomosaics, point clouds, terrain models, and $3 \mathrm{D}$ textures. NodeODM is the most user-friendly tool in the OpenDroneMap ecosystem, providing a lightweight REST API to access and parameterize the software using any desktop browser.

Node-ODM uses Docker to create a Linux-based virtual machine on Windows 10. The first step is to download and install Docker Desktop for Windows at this link: https://www.docker.com/products/docker-desktop. Additional installation details are provided in section 2.3 .

The GRL team created a NodeODM Manager to facilitate setting up, running, and maintaining NodeODM in Docker. The NodeODM Manager creates a desktop shortcut to a series of Windows batch files that will perform a one-time pull of NodeODM from DockerHub, start the NodeODM Docker container, and open a web browser session to start processing (figure 10).

The user should first run the Start OpenDroneMap Docker Instance tool, which will start NodeODM within an instance of Docker hub. Then the main tool is the Open OpenDroneMap Browser Instance, which opens the default internet browser (for example, Google Chrome). The user is taken to a local interface and directed to add UAS imagery for processing. Initial outputs include color-balanced orthomosaics, point clouds, and much more, as shown directly within an interface similar to Figure 10. 
Figure 10. NodeODM Manager displaying local REST API session in Chrome browser.

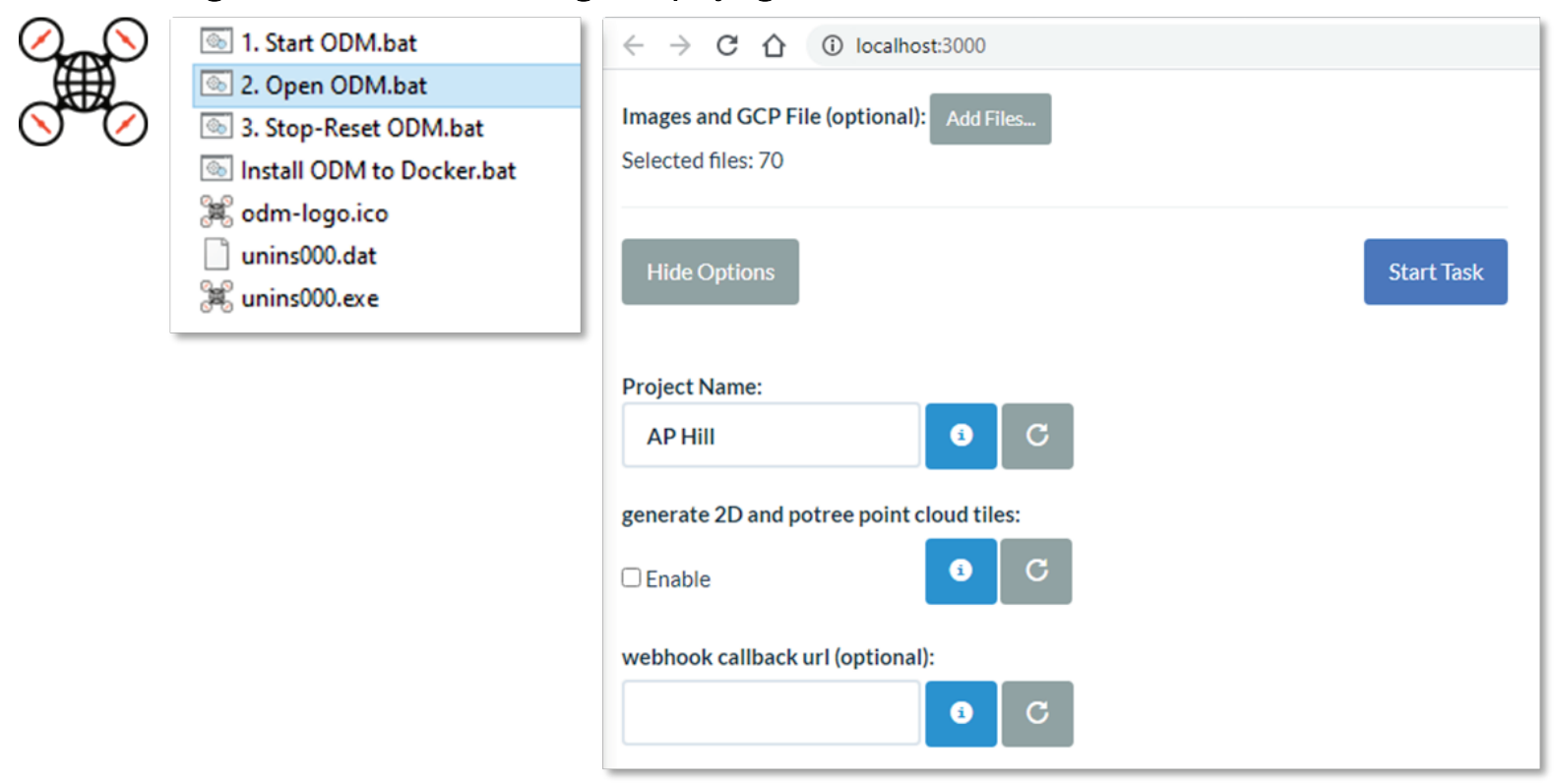

The NodeODM web browser interface offers default processing and output options in addition to user-defined products. An orthomosaic is the standard product; however, other output options include point cloud development, processing, and formatting as well as DTM and DSM layers defining a custom spatial resolution and $3 \mathrm{D}$ texturing. The examples below show an orthomosaic DSM, and an RGB-colored point cloud developed in NodeODM (Figures 11-12). 
Figure 11. Final orthomosaic and digital surface model produced by NodeODM (Fort AP Hill). Top right: colorized DSM; bottom right: wetland zone.

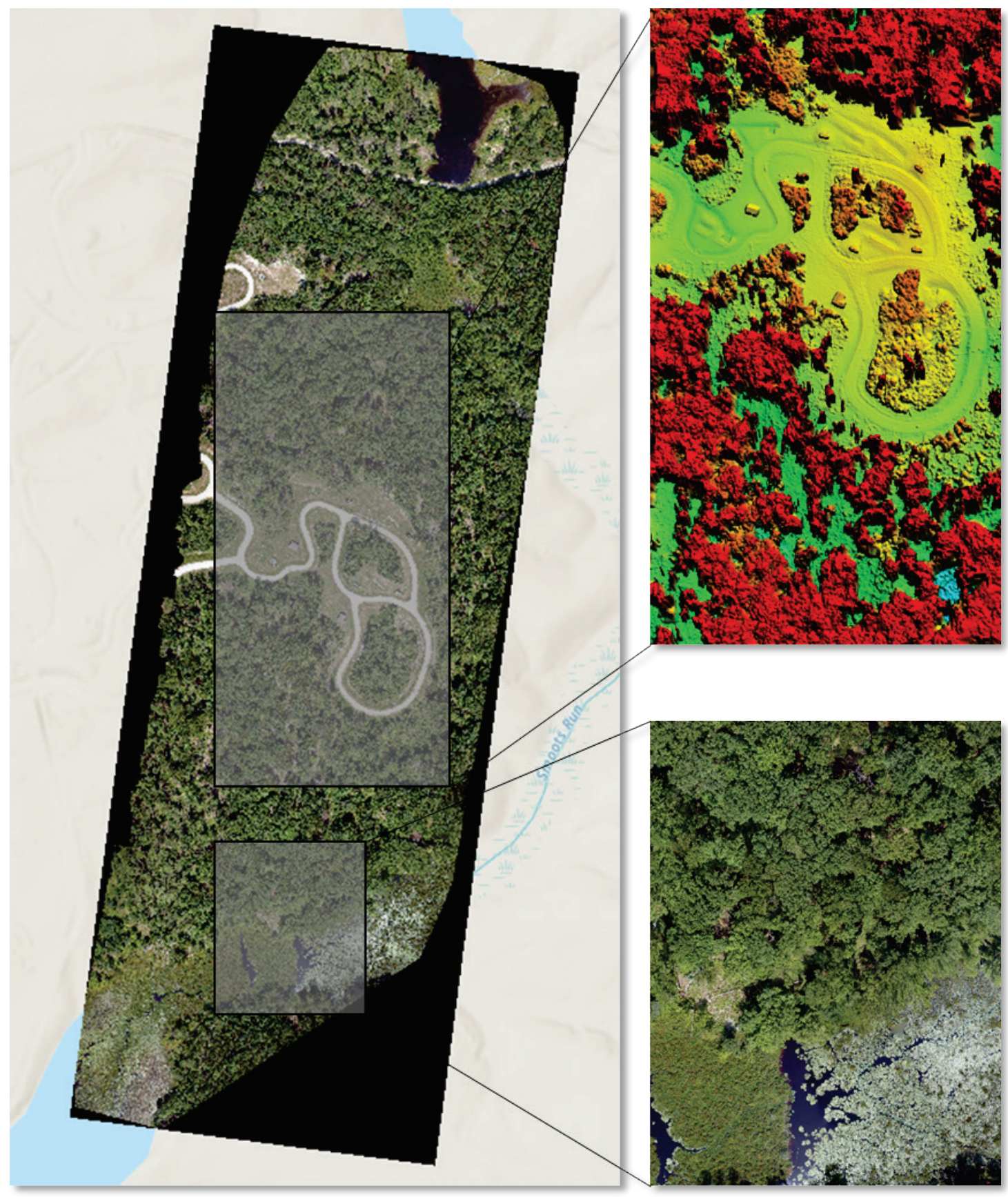


Figure 12. Colorized point-cloud image displayed by height.

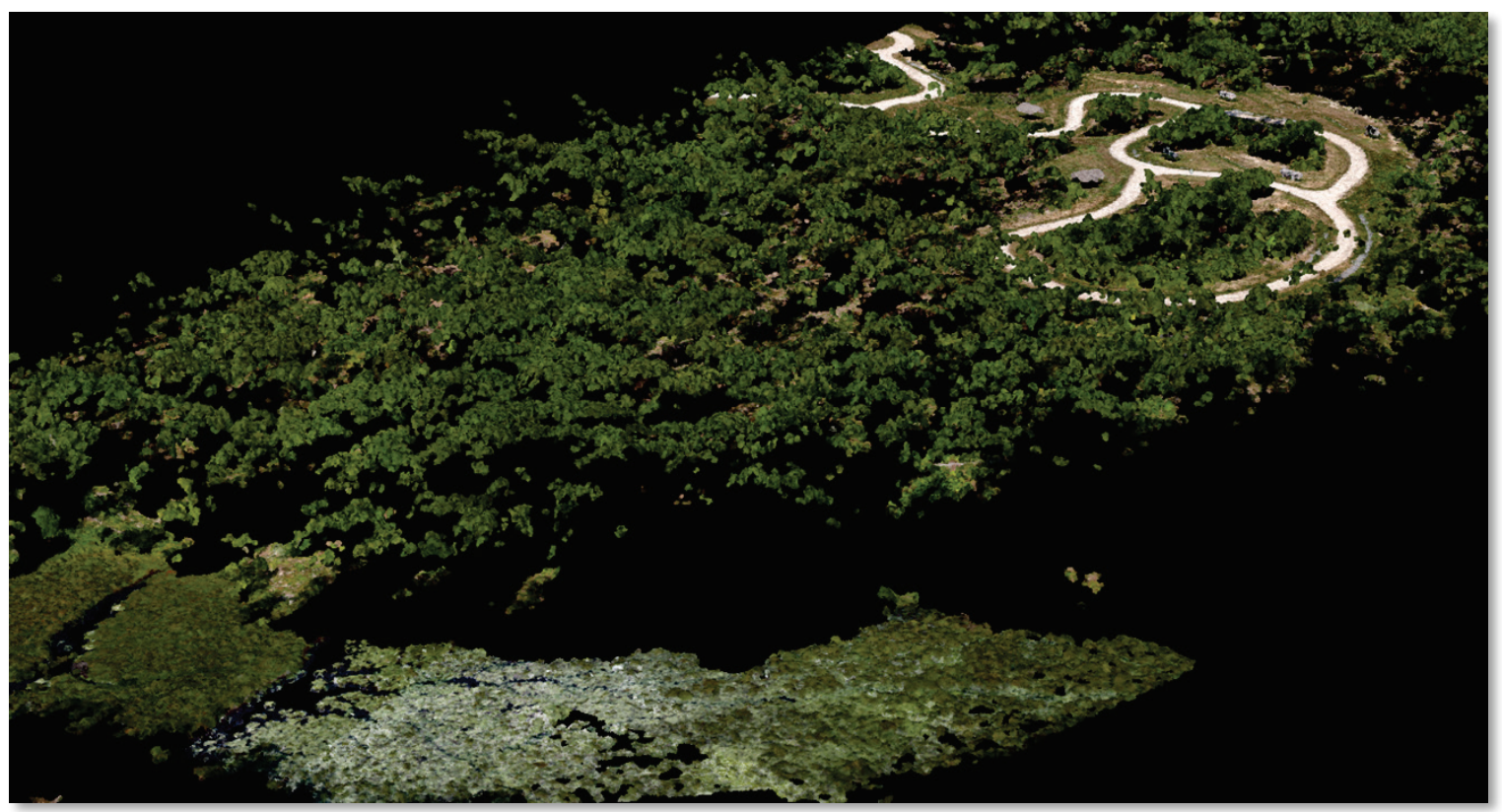

\subsection{Satellite Imagery Tools}

The EMRRP MERIT satellite imagery tools will complete all major steps of satellite data processing and analysis. Within ArcGIS Pro, the tools take the user through image acquisition, to image processing, and lastly to decision-support outputs and data analysis. The tools offer a batch processing capability to handle multiple images. Figure 13 provides a visualization of the tool layout in ArcGIS Pro, and Figure 14 provides a conceptual model of the tools. The tools are written using Python 3 and leverage not only open-source Python libraries such as NumPy, gdal, scikit-learn, and Geopandas but also the commercial ArcPy site package and freely available third-party, command-line tools. This capability is wrapped into a custom GUI within ArcGIS Pro, which enables the tools to output directly into ArcGIS for rapid decision-support products. 
Figure 13. Layout of MERIT in ArcGIS Pro showcasing the different sets of tools and associated organization structure.

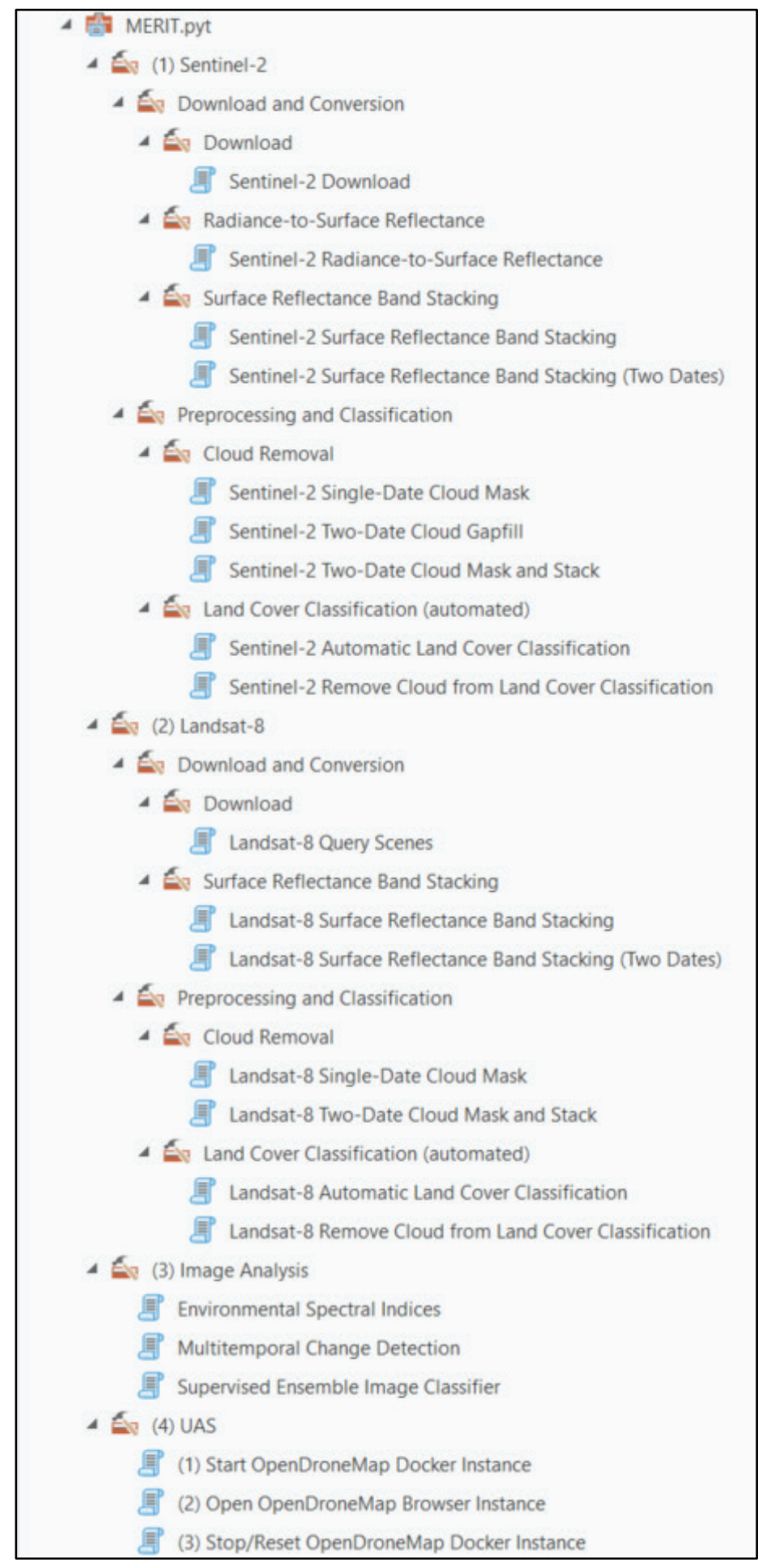


Figure 14. Conceptual model of the satellite image processing and analysis tool suite with an example, likely processing workflow.
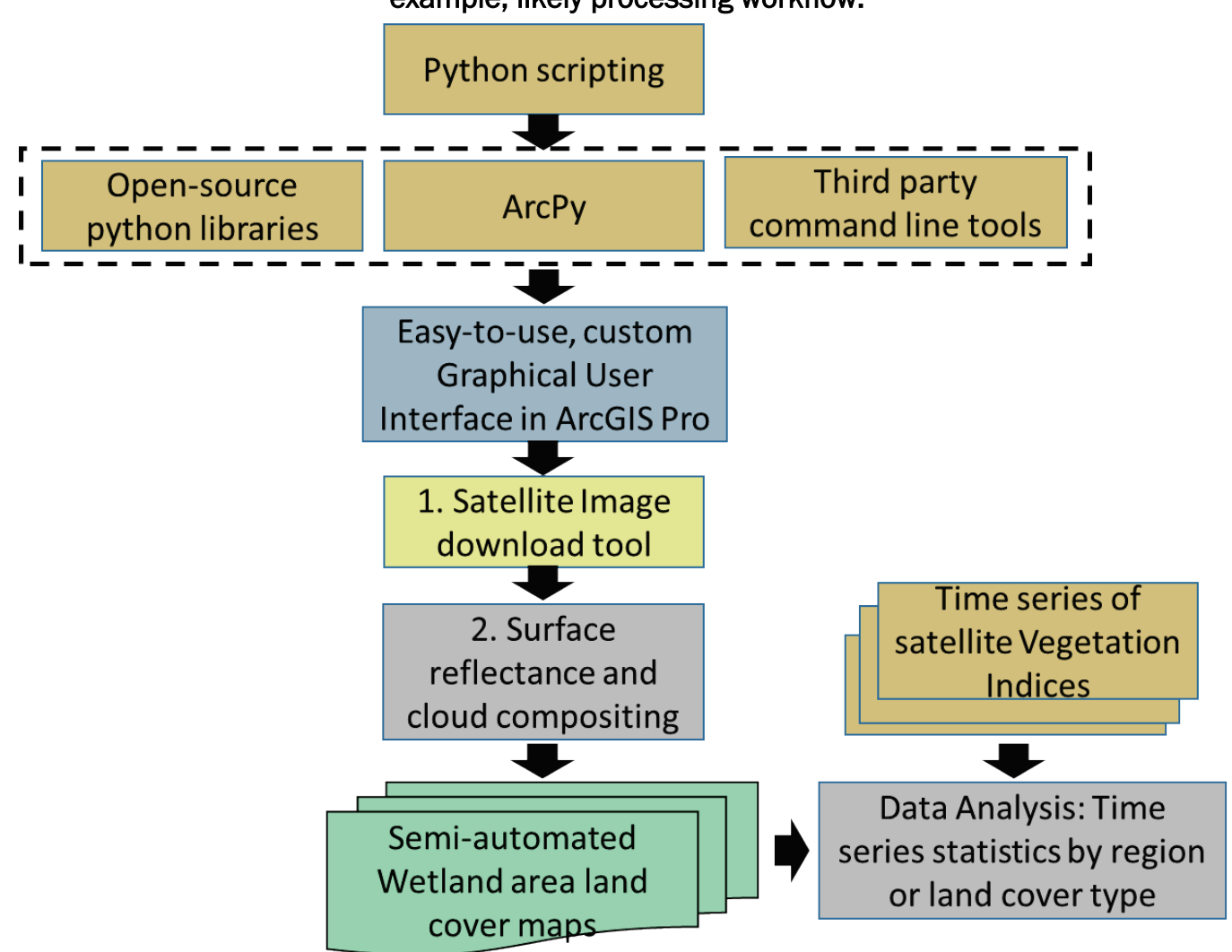

The satellite imagery tools are designed for compatibility with the most prevalent globally available satellite data products: NASA's Landsat 8 and European Space Agency (ESA) Sentinel-2. Landsat 8 provides multispectral imagery at $30 \mathrm{~m}$ nominal spatial resolution with spectral bands of coastal blue, blue, green, red, near infrared, short-wave infrared (SWIR) ( $\times 2$ ), panchromatic, and cirrus. The satellite follows a sunsynchronous, near-polar orbit at an altitude of $705 \mathrm{~km}$ with a 16-day repeat cycle and local equatorial crossing time of 10:00am \pm 15 minutes (https://www.usgs.gov/). The Landsat 8 data are freely available to download in surface reflectance products from the US Geological Survey (USGS) earth explorer website (https://earthexplorer.usgs.gov/), among other sources. ESA's Sentinel-2 consists of two satellites that provide multispectral imagery at 10,20 , and $60 \mathrm{~m}$ nominal spatial resolutions with spectral bands of coastal blue, blue, green, red, red edge $(\times 3)$, near infrared $(\times 2)$, and SWIR $(\times 4)$ (https://sentinel.esa.int/web/sentinel/home). 


\subsubsection{Image Download and Conversion}

\subsubsection{Sentinel-2 image acquisition}

If the user does not already have their own imagery, they can acquire it using the Sentinel-2 Download tool. The tool lets the user enter a date range to search for imagery, specify the maximum permissible cloud coverage in a given image ( $0 \%-100 \%)$, decide how many total images to download, and decide whether to download the top-of-atmosphere product (S2MSIL1C) or the surface reflectance product (S2MSIL2A). Lastly, the user can load or draw their own Area of Interest (AOI) within the tool to specify the area to search for images.

The tool uses geopandas to convert the user-created shapefile into a geoJSON file so that the AOI and user-defined parameters can be used as part of a query to the Copernicus Open Access Hub. This public-facing server of the ESA enables global users to freely download Sentinel-2 imagery directly from the source. Our tool makes a query through this server by leveraging the Python package sentinelsat (version 0.14), which provides a convenient Python API for directly querying the server.

The GIS user should note that typically Sentinel-2 surface reflectance imagery greater than one year old may not be available through this tool. These older images are in cold storage and must be ordered and downloaded on the Copernicus Open Access Hub website. The top-ofatmosphere products are typically available for a longer time, but they must be converted to surface reflectance using MERIT. The download tool will acquire the $20 \mathrm{~m}$ Sentinel-2 data set by default; the $10 \mathrm{~m}$ data set offers fewer spectral bands. An example screenshot of the tool, corresponding customized ArcGIS tool messages, and downloaded image are shown in Figure 15. 
Figure 15. Screenshot of the image acquisition tool that directly downloads imagery in batches from ESA public-facing servers over a user's AOI. Tool message output is shown at the top right, highlighting that the user is informed of each step in the process as well as any errors. We recommend $20 \mathrm{~m}$ imagery because of increased spectral versatility and compatibility with automatic wetland classification tools.

\section{Sentinel-2 image downloading integrated directly into ArcGIS Pro}

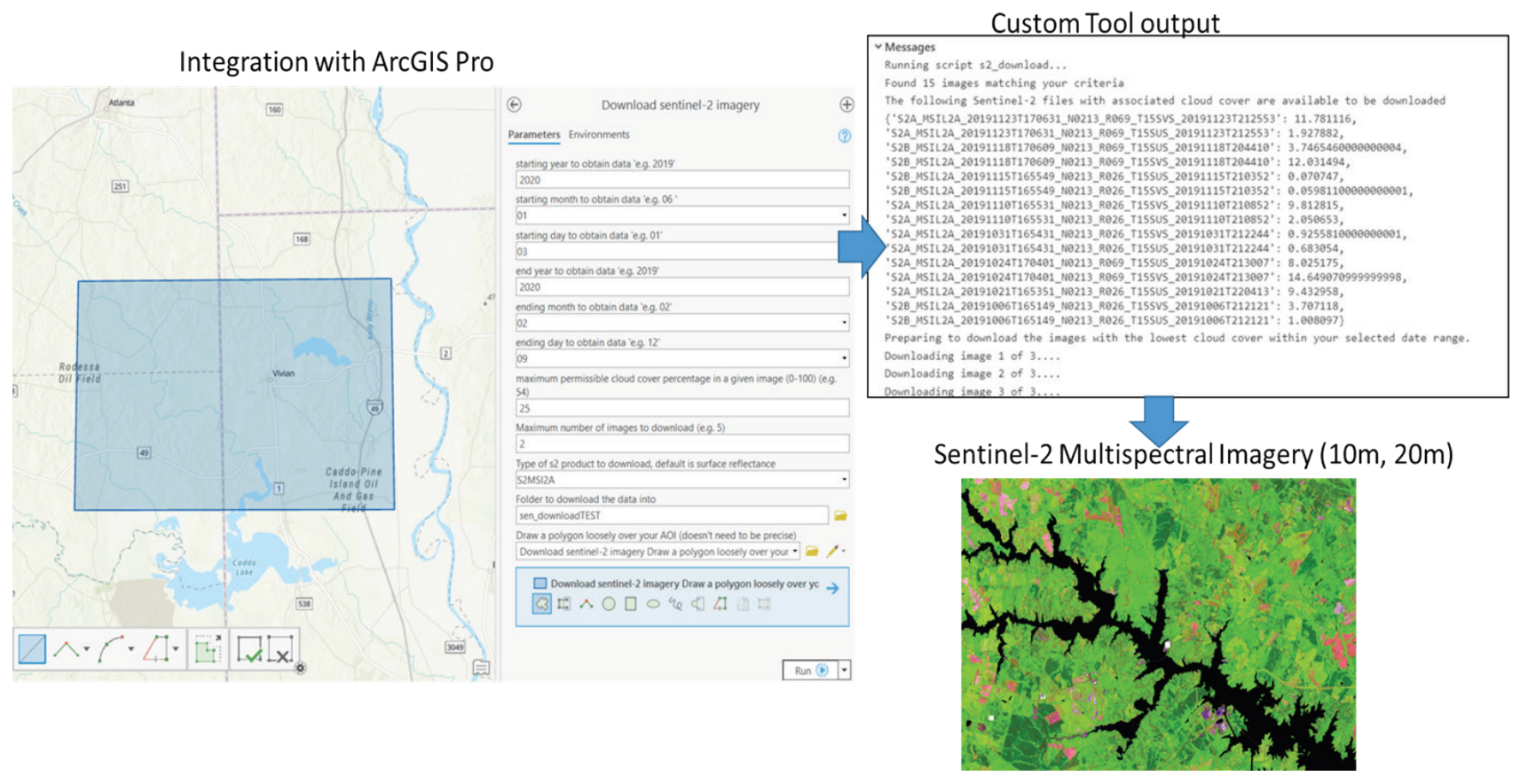

\subsubsection{Conversion of Top-of-atmosphere Radiance to Surface Reflectance}

It is critical to convert satellite imagery into surface reflectance. Surface reflectance products have mitigated the influence of the Earth's atmosphere on the reflectance product and thereby yield an earth surface reflectance product, typically expressed in radians per unit area $\left(\mathrm{rad} / \mathrm{m}^{2}\right.$ or $\mathrm{cm}^{2}$ ). In contrast, top-of-atmosphere reflectance products include the atmospheric effects as seen from space, where the image is collected. A user will not be able to accurately monitor a study site over time or collect representative spectral measurements without first converting to surface reflectance. Currently, the $6 s$ vectorial algorithm, originally designed for Moderate Resolution Imaging Spectroradiometer (MODIS) imagery, is often used on Sentinel-2 and Landsat 8 imagery with modifications that exploit the coastal aerosol bands (Vermote et al. 2016). The $6 S$ vectorial algorithm is a coupled, atmosphere-surface, physical-based model that accounts for the scattering of light in the atmosphere because of clouds, aerosols, and other sources. (Kotchenova et al. 2006). 
The Satellite MERIT tools use the Sen2Cor command-line tools for conversion to surface reflectance. Similar to $6 \mathrm{~S}$, Sen2Cor uses radiative transfer modeling, cirrus cloud correction, aerosol optical thickness, water vapor, elevation, and other factors to approximate surface reflectance (Main-Knorn et al. 2017). This processor is easily implementable and generally accepted by the scientific community but has been found to overestimate surface reflectance in comparison to ground-reference data (Li et al. 2018).

The EMRRP MERIT tool uses Sen2Cor version 2.8 and follows a loosely coupled model where the Sen2Cor processor is called using Python within the ArcGIS Pro tool. The tool lets the user input a folder containing multiple Sentinel-2 $\mathrm{L} 1 \mathrm{C}$ files as well as allowing the user to select the output spatial resolution to process ( $10 \mathrm{~m}$ or $20 \mathrm{~m}$ ). We recommend the $20 \mathrm{~m}$ resolution, as it offers additional spectral bands compared to the 10 $\mathrm{m}$ imagery, and is the only resolution compatible with the automatic landcover classification tool. Figure 16 provides a high-level overview of the tool integration.

For Landsat 8, the user will use the Landsat 8 image acquisition tools to directly download surface reflectance-corrected imagery from the USGS server. Thus, there is no need for a conversion tool. 
Figure 16. High-level conceptual model showing how MERIT takes as input a folder of multiple Sentinel-2 images. Then the ArcGIS Pro tool calls the Sen2Cor atmospheric correction tool and converts the image to surface reflectance, as shown in the bottom two images.
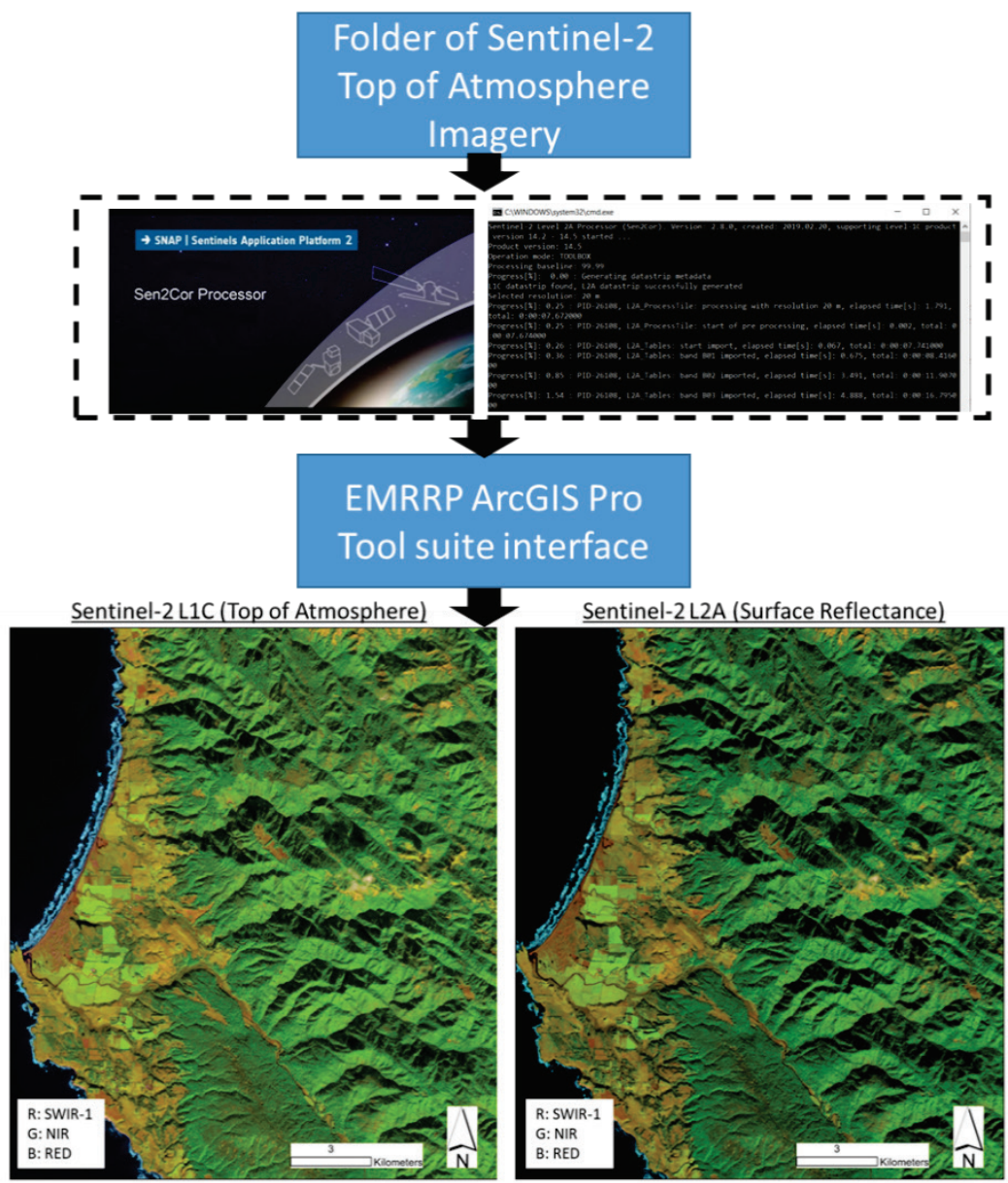

\subsubsection{Landsat 8 image acquisition}

The Landsat-8 Query Scenes tool hastens the image acquisition and downloading process for the user. This tool resembles the Sentinel-2 image acquisition tool but has several important differences. The user will draw a polygon covering the approximate area of interest. Next, the user specifies a date range, cloud cover maximum, and total number of images to download. The tool differs from the Sentinel-2 tool because it will save the product ID of each of the selected Landsat 8 scenes to a CSV file. This information is queried from the USGS EarthExplorer website, a data repository for all Landat 8 imagery. The tool interacts with the USGS site using the landsatxplore python API.

Below are the necessary steps to acquire the imagery: 
1. After runing the tool and obtaining a .CSV file with the Landsat 8 collection 1 product IDs, navigate to the EarthExplorer website at https://earthexplorer.usgs.gov/order/index/ and login with username and password.

2. Select the File Upload option of Landsat Product ID List. Add the .CSV file from the File Selection option, select a file list name, and then click the green Upload File button at the top.

3. Scroll to the bottom and locate the drop-down menus with Select Order Type and Select List titles. Choose the newly created list of Landsat products to download and choose whether to use the bulk download function or the on-demand function to acquire the images.

4. Click the New Order From List button, below which is a list of Landsat scenes to order.

5. Lastly, click the Submit Product Selections button to finalize the image order and follow instructions from there.

\subsubsection{Surface Reflectance Band Stacking}

The surface reflectance band-stacking tools combine all of the separate raster band files into a single image stack. This consolidation allows subsequent analysis and processing with other MERIT subtools and other ArcGIS Pro tools. There are two sets of tools provided, one set for Sentinel2 and another for Landsat 8.

For the Sentinel-2 tool, after running the radiance to surface reflectance tool (if the user did not already acquire surface reflectance imagery), the user need only to select their .SAFE folder containing Sentinel-2 imagery, the resolution (10 m, $20 \mathrm{~m}$ ), and an output file name. Note that we recommend the $20 \mathrm{~m}$ resolution for most cases because, unlike $10 \mathrm{~m}$, it contains additional bands like SWIR. Further, $20 \mathrm{~m}$ is required for the automated wetland area classification tool. Regardless of the resolution, the tool will stack the bands in the order they are shown in the corresponding Sentinel-2 data folder. For the recommended $20 \mathrm{~m}$ resolution, the order is

- B2, blue, central wavelength of $490 \mathrm{~nm}$

- B3, green, central wavelength of $560 \mathrm{~nm}$

- B4, Red, Central wavelength of $665 \mathrm{~nm}$

- B5, visible and near infrared, central wavelength of $705 \mathrm{~nm}$

- B6, visible and near infrared, central wavelength of $740 \mathrm{~nm}$

- B7, visible and near infrared, central wavelength of $783 \mathrm{~nm}$

- B8A, visible and near infrared, central wavelength of $865 \mathrm{~nm}$ 
- B11, SWIR, central wavelength of $1610 \mathrm{~nm}$

- B12, SWIR, central wavelength of $2190 \mathrm{~nm}$

- SCL, scene classification, image-derived data layer

There is a separate, but related, tool called Sentinel-2 Surface Reflectance Band Stacking (Two Dates) designed to do the same process; however, it combines imagery from both winter (leaf-off) and summer (leaf-on) acquisitions. The primary purpose of this other tool is to prepare data for an automatic image classification tool, which we discuss in a subsequent section. The tool combines the winter bands first, followed by the summer bands, both in the same order defined above. The SCL band contains information about clouds and pixel quality useful for removing cloudy pixels with subsequent MERIT image-processing tools.

For the Landsat 8 Surface Reflectance Band Stacking tool, a single image will be created combining all of the input bands. The user first selects their input folder containing the zipped Landsat 8 file, and then selects the Landat-8 file downloaded from the EarthExplorer website. The user can also run the Landsat-8 Surface Reflectance Band Stacking (Two dates) tool, which combines the winter and summer dates of imagery in preparation for the automatic classification tool.

\subsubsection{Image Pre-processing}

\subsubsection{Single Date Cloud Mask (Landsat 8 or Sentinel-2)}

Clouds are a common issue in multispectral satellite imagery because they obscure observations of the ground. In addition, if they are not masked or removed from imagery, then they may lead to issues while using analysis tools, which could result in something like clouds classified as built-up area. To avoid this misclassification, and to present cleaner data, we offer a cloud mask tool that removes cloud cover from either stacked Sentinel-2 $20 \mathrm{~m}$ imagery by using the included SCL band or $30 \mathrm{~m}$ Landsat 8 surface reflectance imagery using the PIXELQA band.

In order to run the Sentinel-2 tool, the user simply inputs their reflectance stack of imagery (nine bands in one image) and chooses an output file name. Whereas for the Landsat 8 tool, the user inputs their six-band image. 


\subsubsection{Two date Cloud Gapfill (Landsat 8 or Sentinel-2)}

Many regions across the globe experience cloud cover that impacts satellite and airborne observations of land cover, especially in the southern United States, where many wetland monitoring sites are present (Wilson and Jetz 2016). Historical methods to overcome this difficulty relied on using entirely cloud-free image scenes. However, cloud-free scenes are often unavailable. Instead, over the past several years, scientists have leveraged pixel-based compositing. Cloud-free pixels from multiple images are composited into a single, cloud-free or cloud-reduced image. More advanced methods of pixel-based compositing employ parametric weighting schemes that use imagery from an entire year to select the ideal pixel according to the day of year (seasonal suitability), distance from a cloud, or other parameters (Griffiths et al. 2013). Studies mapping landcover type have demonstrated the importance of using a time series of cloud-free composites, with output land cover classifications showing more than $10 \%$ higher overall accuracy as compared with a single date classification (Man et al. 2018).

Our ArcGIS Pro tools allow the user to combine two dates of surface reflectance imagery from either Landsat 8 or Sentinel-2 to generate a cloud-free or cloud-reduced image composite for further analysis. The tool will only work on surface reflectance images, as it requires the pixel quality assurance file or cloud mask that is generated in the surface reflectance process (PIXELQA or SCL file). For best compatibility with Sentinel-2, the user should have a folder containing the Level-2A (L2A) surface reflectance bands produced by Sen2Cor or directly acquired from the Copernicus Open Access Hub. For the Landsat 8 imagery, we recommend the Analysis Ready surface reflectance imagery acquired from the USGS EarthExplorer website or to use our Landsat 8 image downloading tool. An example of cloud compositing is shown in Figure 17.

There are separate tools for Sentinel-2 and Landsat 8, which can be found when opening MERIT in ArcGIS Pro. 
Figure 17. Left-side images contain cloudy Sentinel-2 images. The right-side image is a composite that combines cloud-free pixels from both dates in order to create a cloud-reduced composite for subsequent analysis.
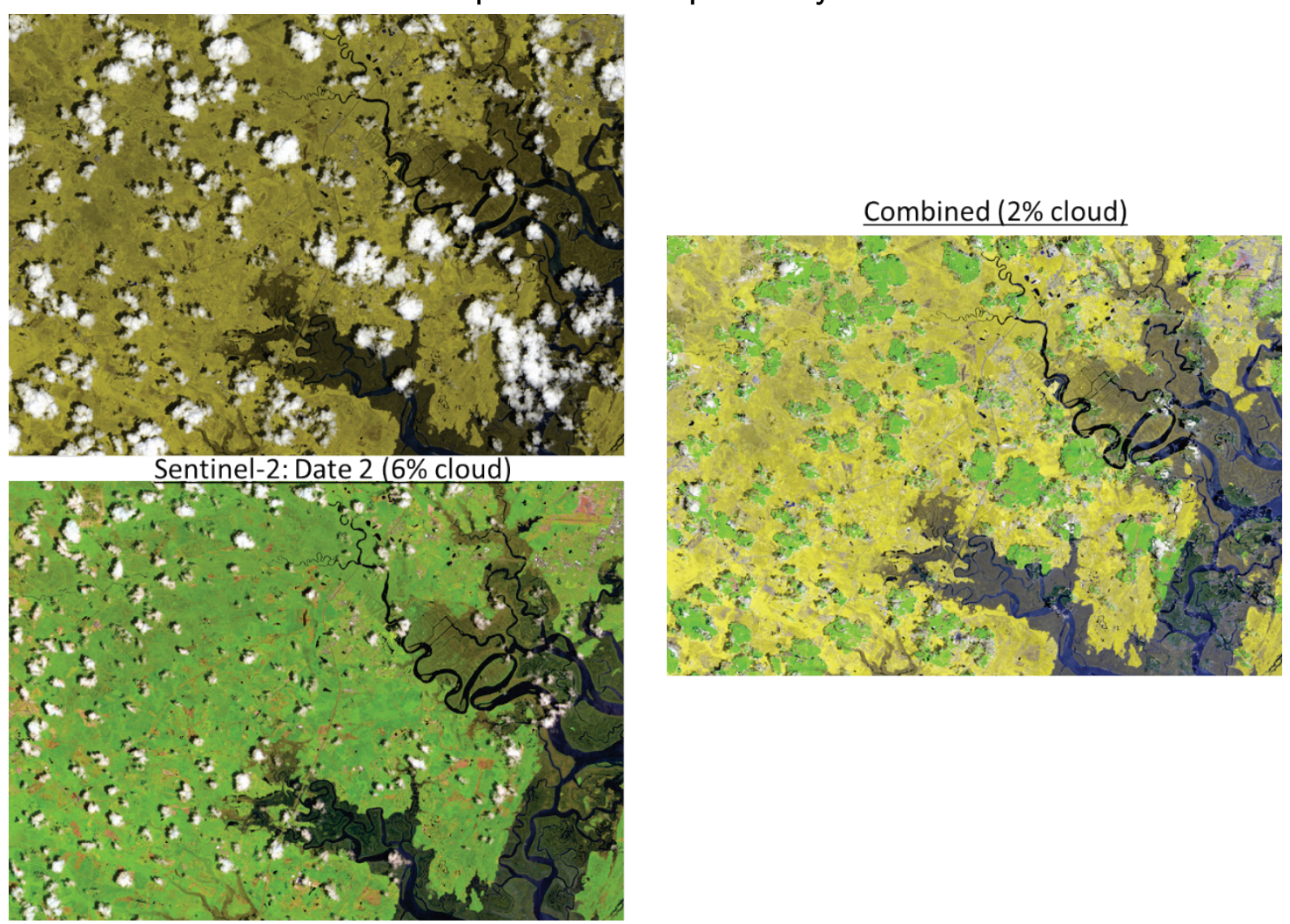

\subsubsection{Image Analysis}

\subsubsection{Batch calculation of environmental indices}

Environmental indices are typically derived from basic ratios between two or more spectral bands of a satellite image. Generally, they create a normalized output that enables comparison of land surfaces over time. The most prevalent indices include the normalized difference vegetation index (NDVI), normalized difference water index (NDWI), normalized difference snow index (NDSI), and soil-adjusted vegetation index (SAVI). These and other indices are often leveraged in environmental monitoring, restoration, and impact studies. For example, the NDWI, NDSI, and NDVI in combination have been used for vegetation phenology monitoring, such as determining date of vegetation green up and snowmelt in arctic regions (Delbart et al. 2005).

Environmental indices are used in many other applications, such as delineating vegetation phenology and quantifying flood damage extent. Indices are also useful as primary or supplemental bands in land-cover 
mapping applications. Multiple studies leverage environmental indices as additional input bands to achieve higher classification accuracies (van Beijma et al. 2014; Torbick et al. 2017; Jin et al. 2018).

The EMRRP MERIT ArcGIS Pro tool includes a batch environmental index tool that enables the user to seamlessly create these simple indices for multiple dates of imagery, without the need to run the tool multiple times. This tool lets the user select from the

- NDVI

- NDWI

- burned area index (BAI)

- WorldView improved vegetation index

- WorldView soil index

- WorldView water index

The user can select one or more indices to be computed. For input to the tool, the user selects one or more raster files to compute the indices for a folder to save all of the output indices and the output data type. The user must select the type of imagery they are using with options available for WorldView and Sentinel-2. The tool will read the image metadata to obtain the correct bands for calculating the indices, and the tool expects that the input imagery are image stacks (for each scene), as opposed to one file for each input band. Example environmental indices calculated using this tool are shown in Figure 18. 
Figure 18. Environmental indices derived from EMRRP MERIT. The top left images shows the custom graphical user interface (GUI) integrated into ArcGIS Pro and highlights that multiple images can be processed.

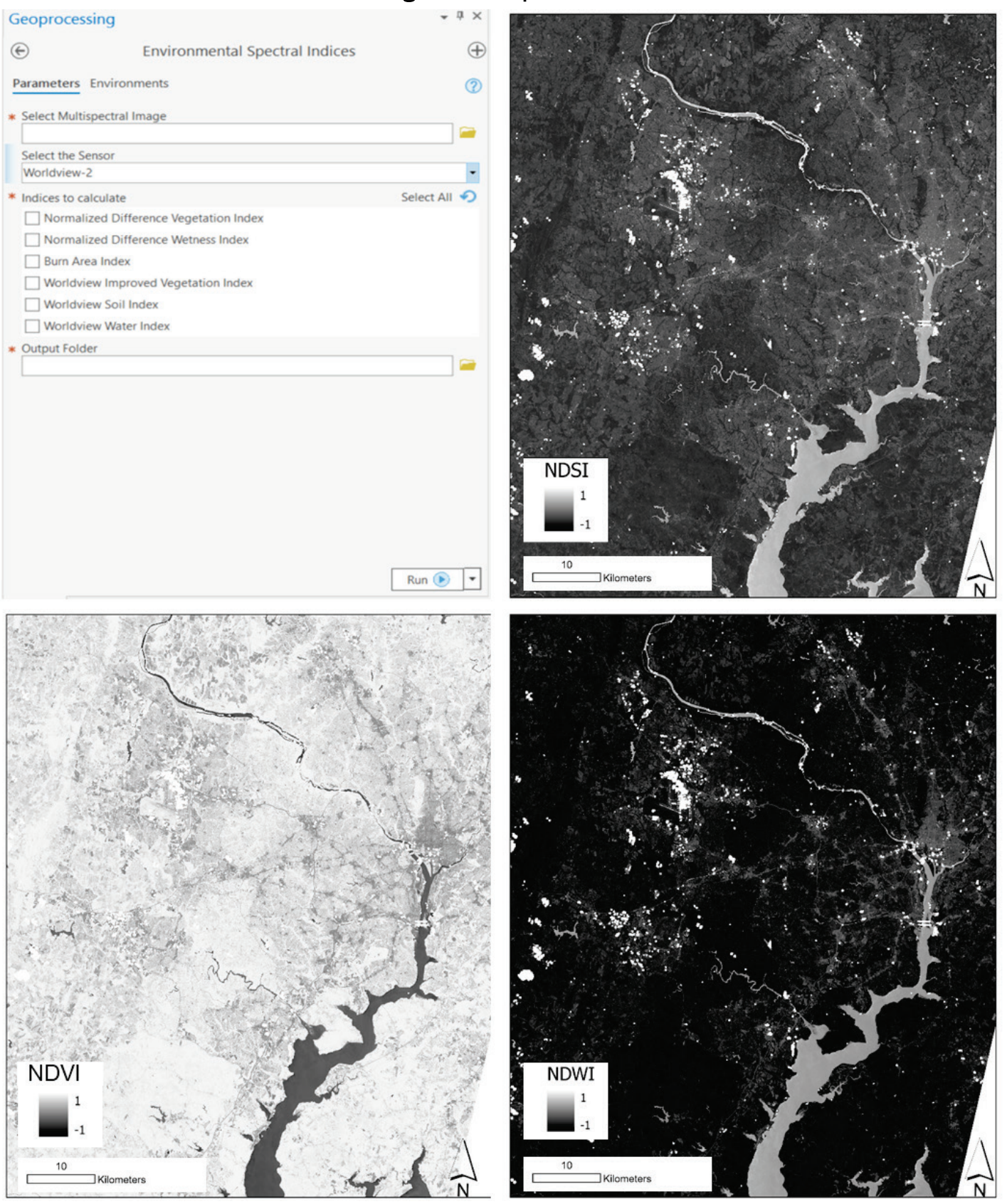

\subsubsection{Supervised Image Classification with Ensemble Classifier}

Image classification in the context of airborne or satellite remote sensing involves separating an image into discrete land surface classes. This classification will most often involve classifying features such as invasive plant or tree mapping, snow cover mapping, mangrove mapping, land use or land cover type mapping, and much more. Image classification 
applications within USACE often include mapping of oil spills, wildland fires, chemical spills, and invasive species (Reif and Theel 2017).

Supervised image classification is the predominant method of segmenting image pixels into discrete classes. User generate points or polygons around features of interest on an airborne or satellite multispectral image. For example, a user would create polygons within mangrove forests, water features, or built-up features as a part of a coastal mapping activity. The spectral band values from the image are then extracted for each point or polygon. These spectral band values and their associated feature labels are used to train the supervised classification algorithm.

There are many types of supervised classification algorithms employed in remote sensing applications. The robust techniques include random forest, support vector machines, neural networks, and stochastic gradient descent. These machine learning algorithms generally outperform userdefined classification schemes, such as maximum likelihood and Euclidean distance discriminant methodologies.

The EMRRP MERIT tool employs a classification scheme that uses three linear classifiers: random forest, support vector machine, and maximum likelihood as implemented in the ArcPy library. The random forest algorithm uses multiple random subsets of training data with an ensemble of best-fit, weak learners (decision trees) to separate the image into distinct classes. The final class is determined according to a majority vote of the individual decision trees (Breiman 2001). The support vector machine operates by creating a hyperplane where the number of dimensions is equal to the number of input spectral bands. The hyperplane generates decision boundaries that separate the image into distinct classes (Sukyens 1999). The maximum likelihood algorithm generally leverages probability distribution functions associated with each class and the probability that a pixel belongs to a given class (Strahler 1980).

Figure 19 provides a workflow model for the ensemble classifier. The user inputs any UAS, airborne, or satellite imagery then selects which output classifiers to use. In order to have an ensemble result of the three supervised classifiers, the user sets the random forest, support vector machine, and maximum likelihood to Yes. The user then selects the corresponding shapefile containing polygons delineating different regions of interest (for example, pine forest, scrubland, grassland). This training 
file should be created and exported using ArcGIS Pro's Training Sample Manager tool. Lastly, the user should select a folder to save any output. The tool will also create a .txt file that describes the tool options that were selected.

Figure 19 also provides an example ensemble map using this tool. The output pixel agreement layer provides the user with an elementary form of map accuracy. It shows the locations where (1) none of the three classifiers agreed on the given pixel's classification, (2) two of the classifiers agreed on the given pixel's classification, and (3) all of the classifiers agreed on the given pixel's classification. The user can leverage this information to repeat the classification with altered training data to improve classification accuracy. For example, a user could create additional training polygons in areas where none of the classifiers agreed and then rerun the tool. This additional training should result in improvement of the thematic accuracy of the final class map. This tool is recommended for classifying an image according to the user's scheme of land cover categories.

Figure 19. Ensemble classifier result for an example six-class, user-created image classification scheme with corresponding pixel agreement map. The agreement map shows the number of classifiers that agree on the resulting land cover type for a given pixel. The conceptual model is shown in the middle.

\section{Supervised Classification Result (Ensemble Classifier)}

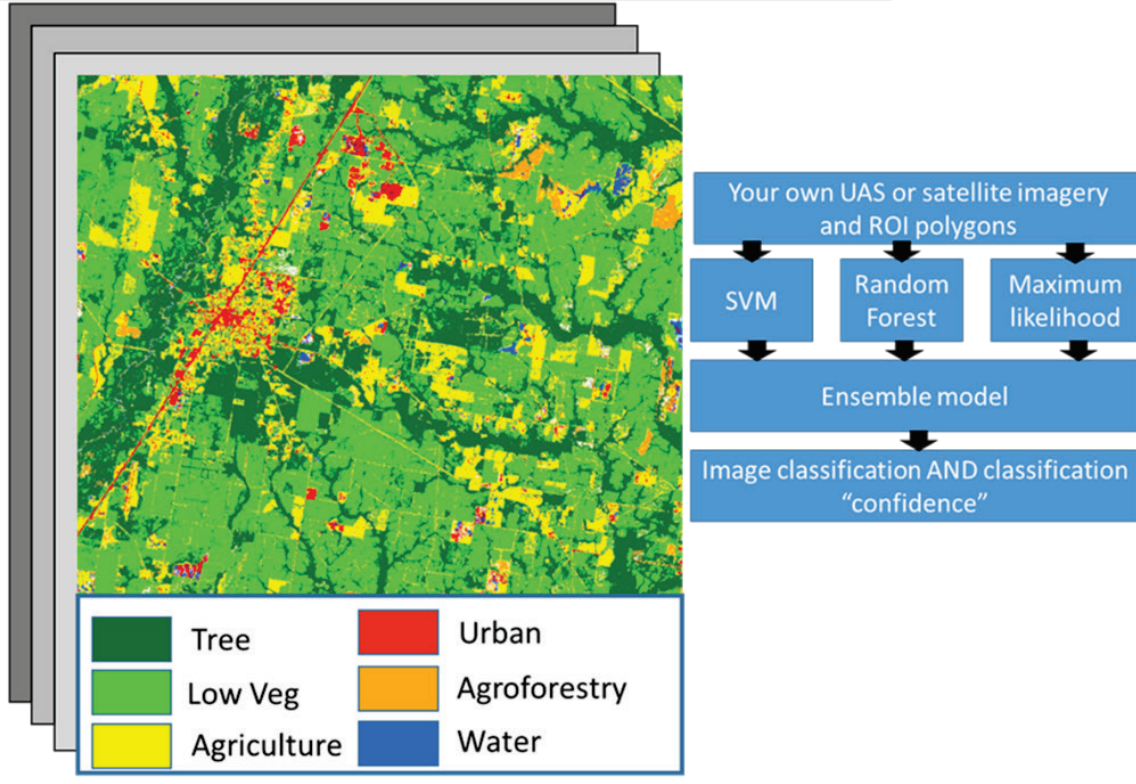

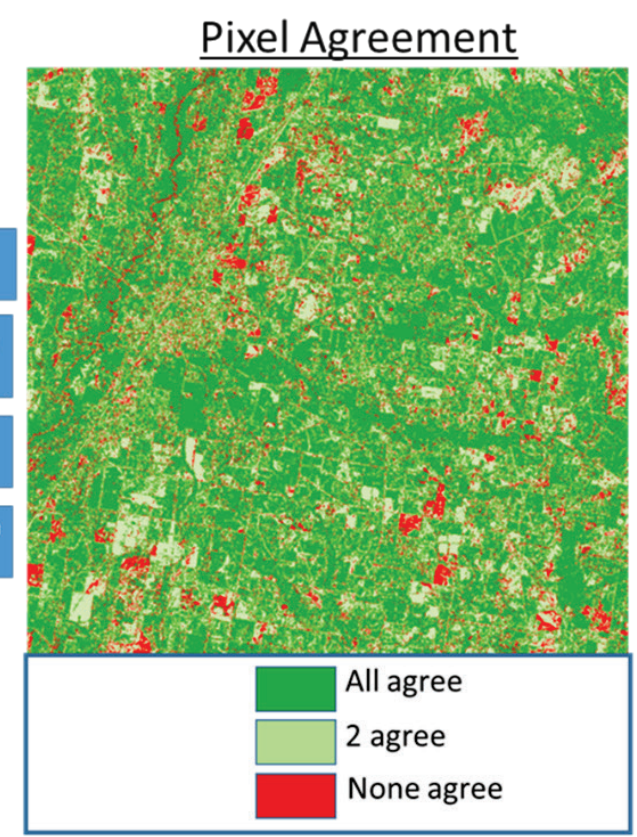




\subsubsection{Zonal Raster Statistics from Time Series}

The Zonal Raster Statistics from Time Series tool handles a time series of data (for example, monthly, daily). Wetland mapping, monitoring, and restoration activities typically require long-term data to track changes over time. For example, the MODIS satellite NDVI monthly product provides globally available observations of NDVI for the period of 2003-present. A user can freely download these data, along with a shapefile of the user's study sites, to monitor the variation of NDVI over time. This variation could show changes in native or invasive vegetation, deforestation, and water levels or flooding.

To use this tool, the user provides an input folder containing raster files with environmental variables such as NDVI (ideally in geotiff format). This folder should not contain extra raster files, or else they may be included in the tool processing. The user also inputs a shapefile that contains the regions, or zones, that delineate the pixels used to calculate the time series zonal statistics. For example, if a user has three sites in a single file, the tool will calculate not only separate statistics for each site but also combined statistics for the sites. Next, the user provides the field name for the regions or zones in the shapefile. Lastly, the user specifies the location in the file name where the temporal information is provided. For example, if the filename is "NDVI2020_05_31processed.tif", the starting character location for the year is 5 and the ending character location is 8 . The user will need to enter this information for month and day as well. The tool was designed for daily data-or at least, data with month, day, and year labels.

Figure 20 provides an example flowchart of this tool. In this example, the one year of monthly MODIS MCD64A1 burned-area data was selected for analysis (the user may select any remote sensing data source). The regions of interest were the states of Texas and California. The tool produces several outputs:

- individual .dbf data tables for each input time step (month in this case)

- combined .dbf table of all time steps

- separate files for monthly, annual averages combined for all zones

- separate files for monthly, annual averages for each individual zone

For visualization purposes, it is recommended that the user export any of the combined .dbf files as comma separated files and create a line graph using the data in a spreadsheet. 
Figure 20. Example possible output for the regional statistics tools. The user inputs a shapefile of their Area of Interest (AOI), a time series of raster data, and outputs corresponding tables that can create temporal visualizations of an area. This example uses burned-area data; however, a variety of raster data can be used.

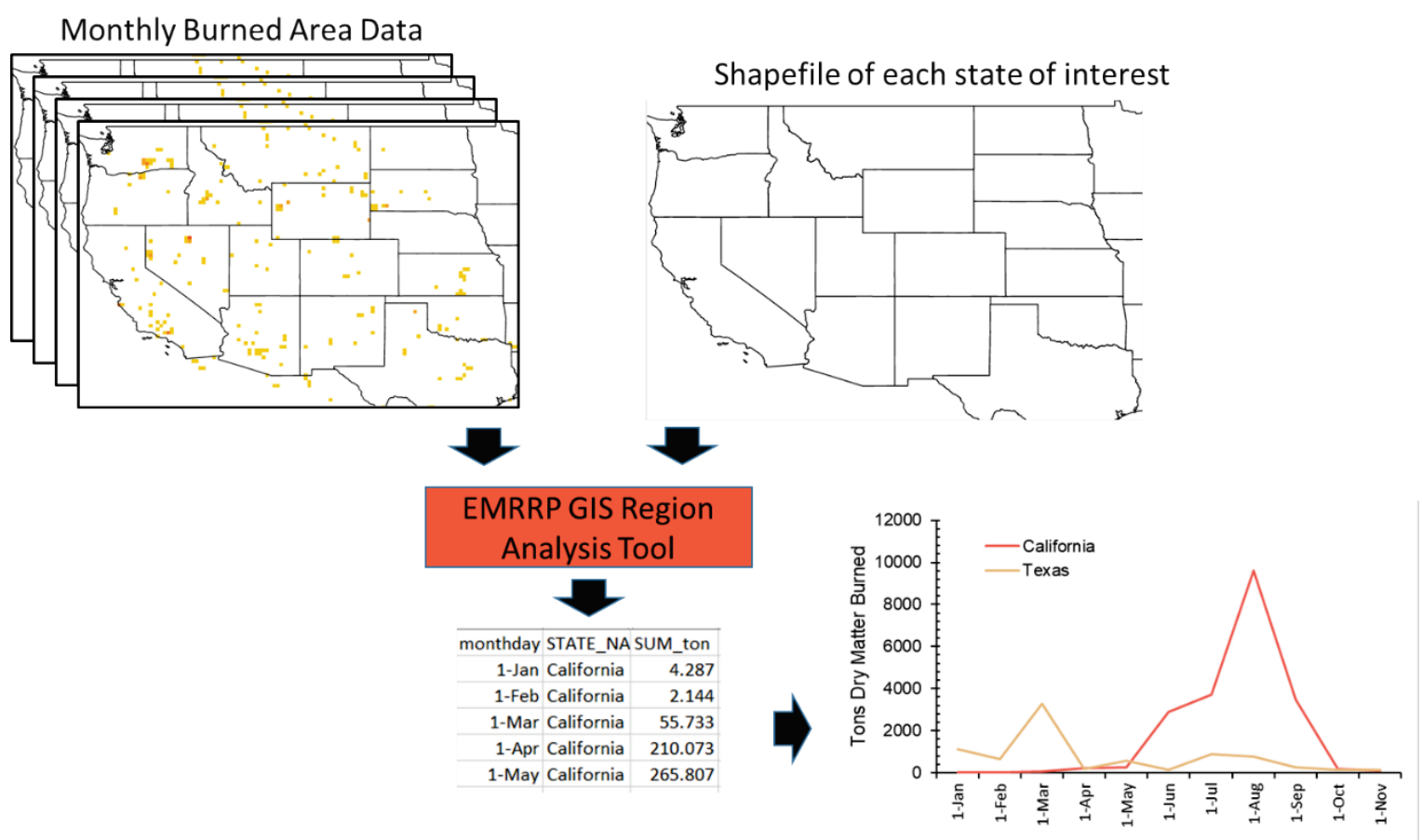

2.2.3.4 Automated wetland area land cover type mapping (Sentinel-2 or Landsat 8)

Land cover is a fundamental variable for characterizing ecosystems, climate, air pollution, flooding, urbanization, and much more. Remote sensing imagery provides repeated and consistent observations over a given location, which can enable monitoring of vegetation phenology, aquatic and terrestrial ecosystems, and changes to the land cover over time. There is a variety of globally available land cover maps created from specific dates in time such as MODIS (500 $\mathrm{m}$ ) land cover available annually between 2001 and 2016, which includes 16 land cover classes according to the International Biosphere-Geosphere Programme classification or the University of Maryland classification with overall accuracy near 75\% (Sulla-Menashe et al. 2019). At the national scale, higher resolution maps are available. For example, the National Land Cover Database (NLCD) provides a 17-class land cover map for years 2001, 2006, 2011, and 2016 based on Landsat time series at $30 \mathrm{~m}$ resolution covering the continental United States (Yang et a. 2018). 
While the aforementioned pregenerated land cover maps with advanced classes are useful for specific dates and for broad analyses, they may prove outdated for a given application. For example, the latest 2016 NLCD map may no longer be accurate for a study conducted in 2020, as land cover frequently changes. Moreover, the broad maps contain many specific classes (for example, 16 classes); however, they have relatively high uncertainty and accuracy issues due to covering broad areas with many classes (Sulla-Menashe et al. 2019).

As a part of the EMRRP MERIT tool suite, a land cover mapping tool was created that leverages user-provided Sentinel-2 or Landsat-8 imagery to automatically generate a land cover type map over wetlands. Supervised training samples were produced across wetland sites in the southern and eastern United States representative of coastal and inland wetland ecosystems (for example, in Arkansas, Florida, Louisiana, Maryland, and Virginia). The training polygons were created separately over Sentinel-2 and Landsat 8 imagery for the following generalized land cover types:

- water

- barren land

- low vegetation

- deciduous trees

- evergreen trees

- herbaceous wetlands

- woody wetlands

- seasonal water

- built up

- croplands

These data were used to train linear support vector machines in Python using scikit-learn (one for Sentinel-2, one for Landsat 8). The classification training data was collected on two dates of imagery-a summer image (May-Sep.) and a winter image (Nov -Mar)-using the surface reflectance bands. The trained models and algorithm were then converted into an ArcGIS Pro Python toolbox so that the end user can run the algorithm as a tool with a custom set of input imagery.

While training the classifiers, $20 \%$ of the samples were withheld at random (stratified according to class) to be used for quantitative accuracy estimation. The remaining $80 \%$ were used to train the classifier. The 
overall thematic accuracy of the output class map was 0.88 as represented by the F-1 score, which is an average of precision and recall. The classspecific F-1 scores were water (0.98), barren land (o.86), deciduous trees (o.89), evergreen trees (0.94), herbaceous wetlands (0.91), woody wetlands (0.89), croplands (0.84), low vegetation (0.89), seasonal water (o.82), and built-up (0.81). The accuracies for the Sentinel-2 model were similar.

To run the automatic classification tool, the user inputs Sentinel-2 or Landsat 8 imagery (composite image containing first winter, then summer bands). The tool then automatically locates the folder that contains the ancillary classification model files provided with the tool. These ancillary files are used for final visualization and classification.

Some data preparation is required before running this tool. Surface reflectance imagery must be acquired directly from NASA, USGS, or ESA, ideally created using the EMRRP MERIT tool. If the user acquired Sentinel-2 L1C product, they must convert it to surface reflectance using EMRRP MERIT. The user should have both leaf-on and leaf-off images covering the same exact spatial extent. Registration and image subsetting may be required. Any snow cover will be misclassified; we recommend snow-free imagery. If cloud cover is an issue, the user may download several images and compute a cloud composite using the MERIT cloud removal tool. Finally, if using Sentinel-2 imagery, the user should run the Sentinel-2 Surface Reflectance Band Stacking (Two Dates) tool, which will create an image stack using the two-date image composite tool, which stacks bands 1-10 from winter and bands 11-20 from summer. The bands must be in the following order: blue (Bo2), green (Bo3), red (Bo4), red edge (Bo5), vegetation red edge (Bo6), red edge (Bo7), near infrared (B8A), SWIR (B11), SWIR (B12), scene classification (SCL) and then followed by the same order for summer bands. The Landsat image stack can be created using the Landsat 8 Surface Reflectance Band Stacking (Two Dates) tool and should create an image composite of 14 bands, seven winter bands first, followed by seven summer bands. The band order should be blue (B2), green (B3), red (B4), near infrared (B5), SWIR (B6SWIR (B7), and PIXELQA band for both winter and summer images. Note that this list excludes the coastal blue band, as it is primarily designed for aerosol or cloud detection. After running the tool using this composite image, the user may then run the cloud masking tools to 
remove any cloud pixels that were misclassified. An example input image and output land cover type map are shown in Figure 21.

Figure 21. Two dates of Sentinel-2 imagery (leaf on, leaf off) are input into the tool to create a wetland area land cover type map. An example output is shown on the right. The user need only input their imagery; no training data is required.
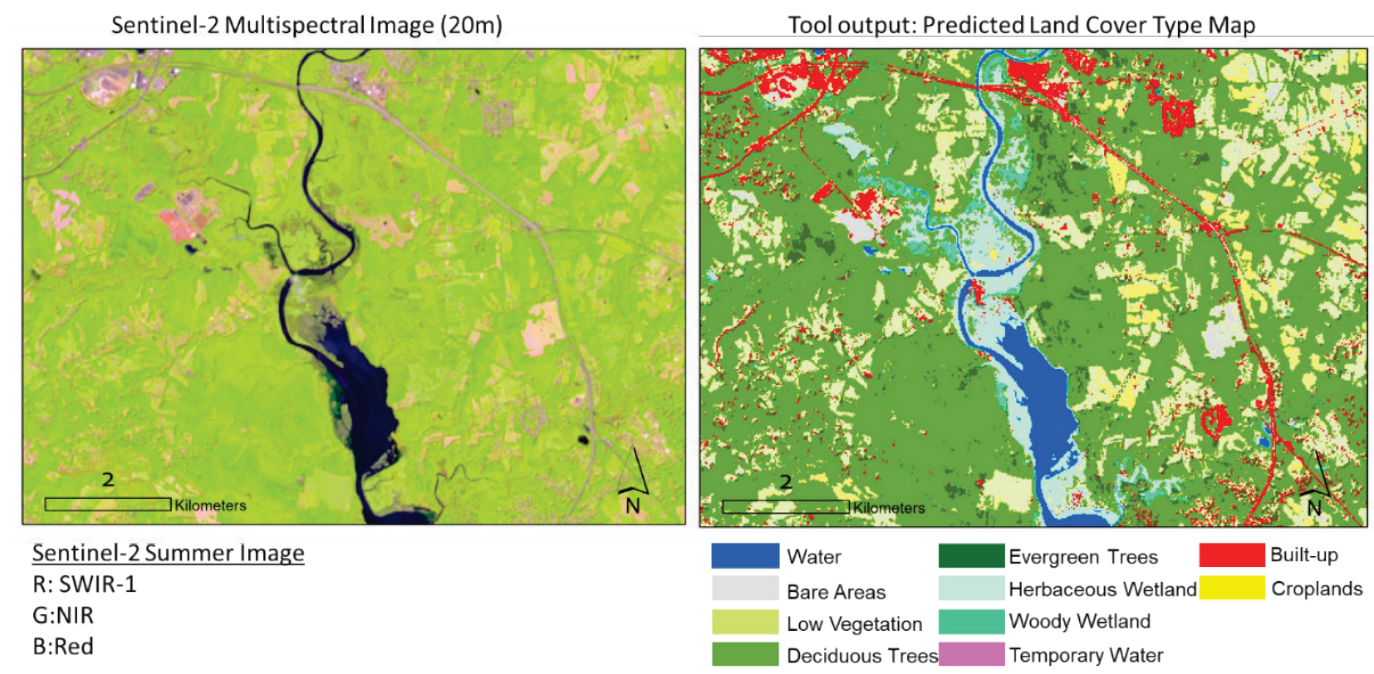

\subsubsection{Multi-temporal Change Detection}

Change detection is a prevalent geospatial technique used in ecological restoration, environmental science, and other land management applications. The general premise of change detection is to input imagery from one date and observe how the landscape changed as compared with a future date of imagery. Oftentimes, an environmental index, such as NDVI or NDWI, is used for computing change detection.

The Multitemporal Change Detection tool provides several options for change detection. The user can choose between absolute, percent, or significant. The first option will output an image with each pixel representing the absolute difference, in image units (for example, digital number, $\mathrm{rad} / \mathrm{m}^{2}$, spectral index value), between the two images. The second option will show the percent change from date one to date two. The third output will show only pixels that changed beyond a user-defined standard deviation from the mean. This tool is designed for continuous data and imagery; it is not designed to quantify change between discrete raster layers, such as thematic maps. An example is shown in Figure 22. 
Figure 22. Absolute change between two dates of NDVI in a wetland area of Virginia. Most change in this image is associated with seasonality.
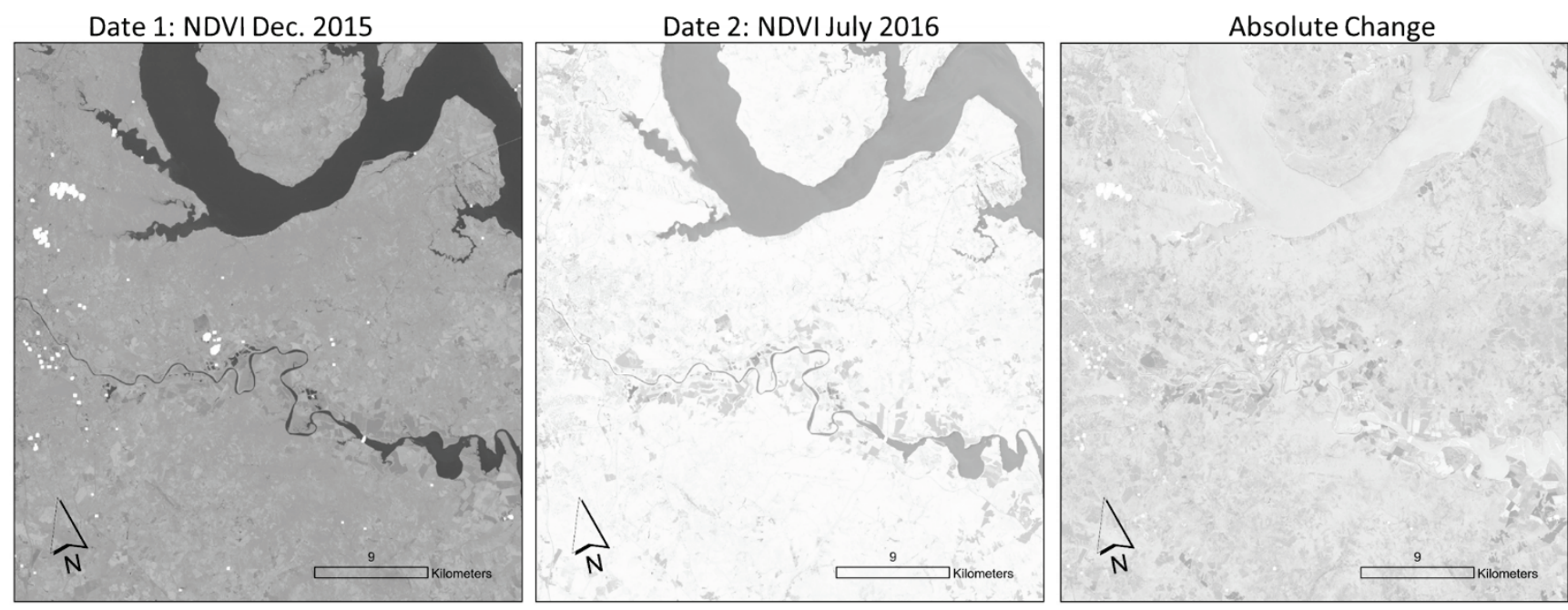

NDVI Dec. 2015

NDVI July 2016

Value

Value 1

Novi Absolute Change

$\mathbf{v}_{-1}$

\subsection{Tool installation and compatibility}

\subsubsection{ArcGIS Pro Tool suite}

The satellite and UAS data processing tools are designed for compatibility with ArcGIS Pro version 2.5 running on a Windows computer. However, the tools may have limited compatibility with other versions, such as 2.3, 2.4, and 2.6. There are two steps to install the MERIT tool suite. First, the user should locate the "MERIT_env_setup_Pro2x.bat" file, right-click it, then choose Run as Administrator. Users unable to run programs as an administrator can simply run it. This file will open a command window and will create a new Python environment called MERIT for use within ArcGIS Pro. It will clone the default ArcGIS Pro Python 3 anaconda environment and then automatically install the necessary libraries and associated dependencies to include joblib (o.15.1), scikit-learn (0.22.1), geopandas (0.6.1), sentinelsat (0.14), and landsatxplore (0.8). This installer will also set the default Python environment within ArcGIS Pro to the newly created MERIT environment.

Next, the user should run the tool installer executable file ("MERIT.exe", right-click, select Run as Administrator) to install the open-source software, including Sen2Cor, NodeODM, and Docker. The tool installer will also add the MERIT tools to your ArcGIS Pro interface. Within ArcGIS 
Pro, locate the Catalog pane. Then navigate to the Toolboxes section, where MERIT will appear. After running the installation tools, restart ArcGIS Pro for the changes to take effect. Also note that there should be at least $500 \mathrm{MB}$ of space available for installing the tools. The "sen2cor.exe" file will install the Sen2Cor command-line tools for surface reflectance correction of Sentinel-2 satellite imagery. Follow the prompts in the installer; it will copy the Sen2Cor files into the Documents folder of the machine in a subfolder called sen2cor. If for any reason, the installation process was incorrect or the user needs to remove MERIT, uninstall the tools using the Add or Remove Programs function of Windows.

\subsubsection{ArcMap Tool suite}

The subset of MERIT designed for ArcMap includes UAS tools organized for compatibility with ArcGIS 10.6 or later. The installation is simpler. The user needs to simply open ArcMap, then navigate to the Catalog window and locate ArcToolbox. Right-click the main toolbox and click Add Toolbox. Then navigate to the MERIT toolkit and select it. The MERIT toolkit now appears in the ArcToolbox. Save this setting so that it persists every time ArcMap is opened by right-clicking ArcToolbox and selecting Settings, then save to default.

\subsubsection{Node-ODM}

NodeODM uses Docker to create a Linux-based virtual machine on Windows 10. First, download and install Docker Desktop for Windows at this link: https://www.docker.com/products/docker-desktop..Next, locate the folder provided with MERIT installation and run the NodeODM Manager Setup. After this, a desktop icon will be created. Click this icon called NodeODM Manager, which will then open a folder containing several files. Open the "README.docx" file, which will take the user through additional steps on how to run the tool successfully. NodeODM is accessed locally on port 3000 using the default internet browser (for example, Mozilla Firefox). 


\section{Conclusion}

This project developed a set of Python-based tools integrated directly into the user-friendly ArcGIS Pro (satellite-based, UAS, and general tools) and ArcMap (UAS tools) software. The tools are designed for USACE district GIS users to perform remote sensing image collection, processing, and analysis in support of monitoring wetlands-as well as any land areas undergoing restoration activities. The UAS tools allow the user to follow a standardized workflow to create colorbalanced orthomosaics, DSM, DTM, and point clouds from UAS imagery. These tools leverage capabilities not directly available within ArcGIS software, such as NodeODM operating within a Dockerized environment.

The satellite-based tools provide a variety of image-processing capabilities that leverage both internal ArcPy functions and third-party scientific software as well as and Python libraries. Some of the available functionalities include bulk downloading of Sentinel-2 imagery, bulk conversion of Sentinel-2 imagery to surface reflectance, calculation of environmental indices, cloud masking of Landsat 8 or Sentinel-2 imagery, cloud compositing, supervised ensemble classification, automated wetland area classification, and regional time series analysis. Many of the tools are designed with batch processing capabilities that enable a user to download or process multiple images without the need to run a tool multiple times. The EMRRP MERIT GIS tool suite is also designed for quick installation, as a self-install file does most of the work. MERIT will be made available with public access for free download through the EMRRP website.

\subsection{Additional Information}

This technical report was prepared by Kristofer Lasko and Sean Griffin, ERDC GRL, with both authors contributing equally. Helpful insight or feedback on the tools was provided by Molly Reif, Glenn Suir, Victor Wilhelm, Brooke Hubbard, and several others. The tools will be made available on https://emrrp.el.erdc.dren.mil// under Resources+, Models section. 


\section{References}

Bedford, B.L., Leopold, D.J. and Gibbs, J.P., 2001. Wetlands ecosystems.

Breiman, L., 2001. Random forests. Machine learning, 45(1), pp.5-32.

Cohen, C.B. and Goward, S.N. 2004. Landsat's Role in Ecological Applications of Remote Sensing. BioScience, Volume 54, Issue 6, Pages 535-545.

Delbart, N., Kergoat, L., Le Toan, T., Lhermitte, J. and Picard, G., 2005. Determination of phenological dates in boreal regions using normalized difference water index. Remote Sensing of Environment, 97(1), pp.26-38.

Dodd, C.K., 1992. Biological diversity of a temporary pond herpetofauna in north Florida sandhills. Biodiversity \& Conservation, 1(3), pp.125-142.

Griffin, S. P., and K. Lasko. 2020. Using Unmanned Aircraft System (UAS) and Satellite Imagery to Map Aquatic and Terrestrial Vegetation. ERDC/GRL TN-20-4. Vicksburg, MS: US Army Engineer Research and Development Center. http://dx.doi.org/10.21079/11681/38086

Griffiths, P., van der Linden, S., Kuemmerle, T. and Hostert, P., 2013. A pixel-based Landsat compositing algorithm for large area land cover mapping. IEEE Journal of Selected Topics in Applied Earth Observations and Remote Sensing, 6(5), pp.2088-2101.

Haarstad, K., Bavor, H.J. and Mæhlum, T., 2012. Organic and metallic pollutants in water treatment and natural wetlands: a review. Water Science and Technology, 65(1), pp.76-99.

Hamilton, S.E. and Friess, D.A., 2018. Global carbon stocks and potential emissions due to mangrove deforestation from 2000 to 2012. Nature Climate Change, 8(3), pp.240-244.

Homer, C., Dewitz, J., Jin, S., Xian, G., Costello, C., Danielson, P., Gass, L., Funk, M., Wickham, J., Stehman, S. and Auch, R., 2020. Conterminous United States land cover change patterns 2001-2016 from the 2016 National Land Cover Database. ISPRS Journal of Photogrammetry and Remote Sensing, 162, pp.184199.

Jin, Y., Liu, X., Chen, Y. and Liang, X., 2018. Land-cover mapping using Random Forest classification and incorporating NDVI time-series and texture: a case study of central Shandong. International Journal of Remote Sensing, 39(23), pp.87038723 .

Kotchenova, S.Y., Vermote, E.F., Matarrese, R. and Klemm Jr, F.J., 2006. Validation of a vector version of the $6 \mathrm{~S}$ radiative transfer code for atmospheric correction of satellite data. Part I: Path radiance. Applied optics, 45(26), pp.6762-6774.

Li, Y., Chen, J., Ma, Q., Zhang, H.K. and Liu, J., 2018. Evaluation of Sentinel-2A surface reflectance derived using Sen2Cor in North America. IEEE Journal of Selected Topics in Applied Earth Observations and Remote Sensing, 11(6), pp.1997-2021. 
Luque, S., Pettorelli, N., Vihervaara, P., and Wegmann, M. 2018. Improving biodiversity monitoring using satellite remote sensing to provide solutions towards the 2020 conservation targets. Methods in Ecology and Evolution. Volume 9, Pages 17841786.

Main-Knorn, M., Pflug, B., Louis, J., Debaecker, V., Müller-Wilm, U. and Gascon, F., 2017, October. Sen2Cor for sentinel-2. In Image and Signal Processing for Remote Sensing XXIII (Vol. 10427, p. 1042704). International Society for Optics and Photonics.

Man, C.D., Nguyen, T.T., Bui, H.Q., Lasko, K. and Nguyen, T.N.T., 2018. Improvement of land-cover classification over frequently cloud-covered areas using Landsat 8 time-series composites and an ensemble of supervised classifiers. International Journal of Remote Sensing, 39(4), pp.1243-1255.

Reif, M.K. and Theel, H.J., 2017. Remote sensing for restoration ecology: Application for restoring degraded, damaged, transformed, or destroyed ecosystems. Integrated environmental assessment and management, 13(4), pp.614-630.

Strahler, A.H., 1980. The use of prior probabilities in maximum likelihood classification of remotely sensed data. Remote sensing of Environment, 1O(2), pp.135-163.

Sulla-Menashe, D., Gray, J.M., Abercrombie, S.P. and Friedl, M.A., 2019. Hierarchical mapping of annual global land cover 2001 to present: The MODIS Collection 6 Land Cover product. Remote Sensing of Environment, 222, pp.183-194.

Suykens, J.A. and Vandewalle, J., 1999. Least squares support vector machine classifiers. Neural processing letters, 9(3), pp.293-300.

Tmusic, G., Manfreda, S., Aasen, H., James, M.R., Goncalves, G., Ben-Dor, E., Brook, A., Polinova, M., Arranz, J.J., Meszaros, J., Zhuang, R., Johansen, K., Malbeteau, Y., Pedrosa de Lima, I., Davids, C., Herban, S. and McCabe, M.F. 2020. Current Practices in UAS-based Environmental Monitoring. Remote Sensing. Volume 12, Pages 1-35.

Torbick, N., Chowdhury, D., Salas, W. and Qi, J., 2017. Monitoring rice agriculture across myanmar using time series Sentinel-1 assisted by Landsat- 8 and PALSAR2. Remote Sensing, 9(2), p.119.

van Beijma, S., Comber, A. and Lamb, A., 2014. Random forest classification of salt marsh vegetation habitats using quad-polarimetric airborne SAR, elevation and optical RS data. Remote Sensing of Environment, 149, pp.118-129.

Vermote, E., Justice, C., Claverie, M. and Franch, B., 2016. Preliminary analysis of the performance of the Landsat 8/OLI land surface reflectance product. Remote Sensing of Environment, 185, pp.46-56.

Wilson, A.M. and Jetz, W., 2016. Remotely sensed high-resolution global cloud dynamics for predicting ecosystem and biodiversity distributions. PLoS biology, 14(3), p.e1002415. 
Yang, L., Jin, S., Danielson, P., Homer, C., Gass, L., Bender, S.M., Case, A., Costello, C., Dewitz, J., Fry, J. and Funk, M., 2018. A new generation of the United States National Land Cover Database: Requirements, research priorities, design, and implementation strategies. ISPRS Journal of Photogrammetry and Remote Sensing, 146, pp.108-123. 


\section{Acronyms and Abbreviations}

\begin{tabular}{|l|l|}
\hline \multicolumn{1}{|c|}{ Acronym } & \\
\hline AOI & Area of Interest \\
\hline DEM & digital elevation model \\
\hline DSM & digital surface model \\
\hline DTM & digital terrain model \\
\hline ENVI & environment for visualizing images \\
\hline ERDC & Engineer Research and Development Center \\
\hline ESA & European Space Agency \\
\hline EMRRP & $\begin{array}{l}\text { Ecological Management and Restoration Research } \\
\text { Program }\end{array}$ \\
\hline EXIF & exchangeable image file format \\
\hline GCP & ground control point \\
\hline GIS & geographic information system \\
\hline GPS & geospatial positioning system \\
\hline GRL & geospatial research laboratory \\
\hline GUI & graphical user interface \\
\hline IDL & interactive data language \\
\hline MODIS & moderate resolution imaging spectroradiometer \\
\hline NDVI & normalized difference vegetation index \\
\hline NDWI & normalized difference water index \\
\hline NLCD & National Land Cover Database \\
\hline ODM & Open Drone Mapper \\
\hline OMW & Ortho Mapping Workspace \\
\hline REST & representational state transfer \\
\hline SAVI & soil-adjusted vegetation index \\
\hline SWIR & Short-Wave Infrared \\
\hline MERIT & Monitoring Ecological Restoration with Imagery Tools \\
\hline TIFF & tagged image file format \\
\hline UAS & unmanned aerial system \\
\hline USACE & US Army Corps of Engineers \\
\hline & \\
\hline & \\
\hline
\end{tabular}




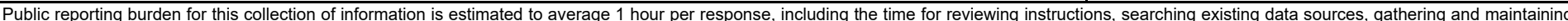

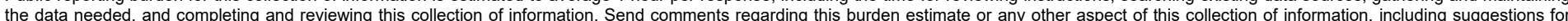

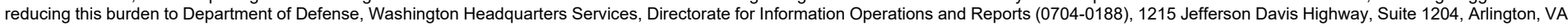

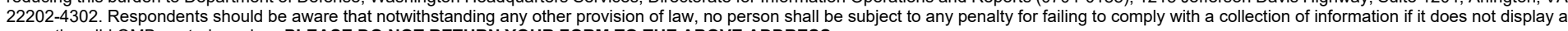
currently valid OMB control number. PLEASE DO NOT RETURN YOUR FORM TO THE ABOVE ADDRESS.
1. REPORT DATE (DD-MM-YYYY) 2. REPORT TYPE
3. DATES COVERED (From - To)

April 2021

Final report

\section{TITLE AND SUBTITLE}

Monitoring Ecological Restoration with Imagery Tools (MERIT): Python-based Decision Support Tools Integrated into ArcGIS for Satellite and UAS Image Processing, Analysis, and Classification

\section{AUTHOR(S)}

Kristofer D. Lasko and Sean P. Griffin

5a. CONTRACT NUMBER

5b. GRANT NUMBER

5c. PROGRAM ELEMENT NUMBER A1120

5d. PROJECT NUMBER 485296

5e. TASK NUMBER

5f. WORK UNIT NUMBER

7. PERFORMING ORGANIZATION NAME(S) AND ADDRESS(ES)

8. PERFORMING ORGANIZATION REPORT NUMBER

Geospatial Research Laboratory

US Army Engineer Research and Development Center

ERDC/GRL TR-21-3

7701 Telegraph Road

Alexandria, VA 22315-3864

9. SPONSORING / MONITORING AGENCY NAME(S) AND ADDRESS(ES)

Headquarters, US Army Corps of Engineers

Washington, DC 20314-1000

10. SPONSOR/MONITOR'S ACRONYM(S)

11. SPONSOR/MONITOR'S REPORT NUMBER(S)

\section{DISTRIBUTION / AVAILABILITY STATEMENT}

Approved for public release; distribution unlimited.

\section{SUPPLEMENTARY NOTES}

\section{ABSTRACT}

Monitoring the impacts of ecosystem restoration strategies requires both short-term and long-term land surface monitoring. The combined use of unmanned aerial systems (UAS) and satellite imagery enable effective landscape and natural resource management. However, processing, analyzing, and creating derivative imagery products can be time consuming, manually intensive, and cost prohibitive. In order to provide fast, accurate, and standardized UAS and satellite imagery processing, we have developed a suite of easy-to-use tools integrated into the graphical user interface (GUI) of ArcMap and ArcGIS Pro as well as open-source solutions using NodeOpenDroneMap. We built the Monitoring Ecological Restoration with Imagery Tools (MERIT) using Python and leveraging thirdparty libraries and open-source software capabilities typically unavailable within ArcGIS. MERIT will save US Army Corps of Engineers (USACE) districts significant time in data acquisition, processing, and analysis by allowing a user to move from image acquisition and preprocessing to a final output for decision-making with one application. Although we designed MERIT for use in wetlands research, many tools have regional or global relevancy for a variety of environmental monitoring initiatives.

$\begin{array}{lll}\text { 15. SUBJECT TERMS } & \text { Remote sensing } & \text { Geographic information systems } \\ \text { Restoration ecology - Monitoring } & \text { Geospatial data } & \\ \text { Environmental management } & & \end{array}$

\section{SECURITY CLASSIFICATION OF:}

\section{a. REPORT}

UNCLASSIFIED

b. ABSTRACT
UNCLASSIFIED

\begin{tabular}{c|c}
$\begin{array}{c}\text { 17. LIMITATION } \\
\text { OF ABSTRACT }\end{array}$ & $\begin{array}{c}\text { 18. NUMBER } \\
\text { OF PAGES }\end{array}$ \\
SAR & 48
\end{tabular}

19a. NAME OF RESPONSIBLE PERSON

19b. TELEPHONE NUMBER (include area code) 